Pacific

Journal of

Mathematics

HECKE ALGEBRAS AND SEMIGROUP CROSSED PRODUCT $C^{*}$-ALGEBRAS

B. BRENKEN 


\section{HECKE ALGEBRAS AND SEMIGROUP CROSSED PRODUCT $C^{*}$-ALGEBRAS}

\section{B. BRENKEN}

For an almost normal subgroup $\Gamma_{0}$ of a discrete group $\Gamma$, conditions are given which allow one to define a universal $C^{*}$-norm on the Hecke algebra $H\left(\boldsymbol{\Gamma}, \boldsymbol{\Gamma}_{0}\right)$. If $\boldsymbol{\Gamma}$ is a semidirect product of a normal subgroup $N$ containing $\Gamma_{0}$ by a group $G$ satisfying some order relations arising from a naturally defined subsemigroup $T$, and if the normalizer of $N$ is also normal in $\boldsymbol{\Gamma}$, then a presentation of $H\left(\boldsymbol{\Gamma}, \boldsymbol{\Gamma}_{0}\right)$ is given. In this situation the $C^{*}$-completion of $H\left(\boldsymbol{\Gamma}, \boldsymbol{\Gamma}_{0}\right)$ is $*$-isomorphic with the semigroup crossed product $C^{*}$-algebra $C^{*}\left(N / \Gamma_{0}\right) \rtimes T$.

In their paper introducing a number theoretical model of a quantum statistical system exhibiting a phase transition with symmetry breaking, Bost and Connes introduce the notion of an almost normal subgroup $\Gamma_{0}$ of a discrete group $\Gamma$, along with the associated Hecke algebra $H\left(\Gamma, \Gamma_{0}\right)$ and its reduced $C^{*}$-algebra completion $C_{r}^{*}\left(\Gamma, \Gamma_{0}\right)([\mathbf{B C}])$. They also provide a presentation of the Hecke algebra in the context of the specific almost normal subgroup they consider in their model. A connection between these relations and some relations occurring in a stable $C^{*}$-algebra associated with certain examples of dynamical systems described in $[\mathbf{B}]$ provided the motivation for considering the Hecke algebras further.

An overview of the structure of the paper follows. After some preliminaries on almost normal subgroup pairs $\left(\Gamma, \Gamma_{0}\right)$ we introduce a fundamental semigroup $\mathcal{T}$ in the group $\Gamma$, which contains the normalizer $N_{\Gamma_{0}}$ of $\Gamma_{0}$. A basic representation of this semigroup as isometries in the convolution Hecke algebra $H\left(\Gamma, \Gamma_{0}\right)$ is described. In the presence of a normal subgroup $N$ of $\Gamma$ containing $\Gamma_{0}$ and contained in $N_{\Gamma_{0}}$, a natural semigroup $C^{*}$-dynamical system occurs which possesses a universal property with respect to $*$-representations of the Hecke algebra.

In the second section we discuss some properties of group partial pre-order relations arising from a subsemigroup of the group in much the same spirit as Nica in $[\mathbf{N}]$. Applying this to our situation, with $\mathcal{T}$ as the subsemigroup of $\Gamma$, and introducing a notion of solvable least upper bounds, we obtain some conditions allowing a definition of a universal $C^{*}$-norm on the Hecke algebra. Assuming some more structure for the pair $\left(\Gamma, \Gamma_{0}\right)$, namely that $\Gamma$ is an extension of a normal subgroup $N$ containing $\Gamma_{0}$, we obtain that 
the $C^{*}$-completion of the Hecke algebra is a quotient of a semigroup crossed product $C^{*}$-algebra.

The main focus of Section 3 is to obtain an identification of the $C^{*}$ completion of the Hecke algebra and the semigroup crossed product $C^{*}$ algebra if the group $\Gamma$ is a semidirect product of a normal subgroup $N$ containing $\Gamma_{0}$ and a subgroup $G$ which is both upward and downward directed. We also assume that solvable least upper bounds exist in the fundamental subsemigroup. This identification is proved by patterning our arguments after those of Bost and Connes to obtain a presentation of the Hecke algebra. A crucial role is played by the covariance relation Nica isolated in $[\mathbf{N}]$. Once the identification is established, we can conclude that some of the relations were superfluous, as they are unnecessary in a presentation of the semigroup crossed product $C^{*}$-algebra.

Section 4 lists some examples pertaining to various stages in the structure of assumptions needed in the course of the paper.

Once a semigroup crossed product structure is available for these Hecke algebras, simplifications in the dynamical structure of the Hecke algebras can occur. For example, it is hoped that the study of the KMS state simplex and phase transitions under a one parameter automorphism group of the algebra, as first explored by Bost and Connes, can be extended to the other examples of Section 4.

As this paper was being prepared for submission we heard that results pertaining to Examples 4.1 and 4.5 discussed here were also being obtained in joint work of Arledge, Laca and Raeburn. The methods and approach employed are however different.

Notation. If $X$ is a set, $|X|$ denotes the cardinality of $S$. For sets $X$ and $Y, X \cong Y$ means that $X$ and $Y$ are isomorphic as sets. If $\mathcal{A}$ is a set of transformations of a set $X$, then $\mathcal{A} x=\{a(x) \mid a \in \mathcal{A}\}$ for $x \in X$. If $H$ is a subgroup of group $G$, write $H \leq G$. Let $N_{H}$ be the normalizer of $H$ in $G$, and if $H$ is normal in $G$ write $H \unlhd G$. Also $[g]$ is the left coset $g H$ in $G / H,(g \in G)$, and the index of $H$ in $G$ is $(G: H)=|G / H|$. For $g \in G, \operatorname{ad}(g)$ is the group automorphism of a normal subgroup $H$ defined by $h \rightarrow g h g^{-1},(h \in H)$. The unit element of a group or semigroup is $e$. The natural numbers with zero, a semigroup under addition, are denoted by $\mathbb{N}$, while $\mathbb{N}^{\times}$denotes the non-zero elements of $\mathbb{N}$, an abelian semigroup under multiplication. If $R$ is a ring, $R^{\times}$denotes the non-zero elements of $R$. For $d \in \mathbb{N}^{\times}, \mathfrak{M}_{d}(R)$ denotes the $d \times d$ matrices with entries in $R$. For $F \in \mathfrak{M}_{d}(R), F_{t}$ denotes the transpose matrix.

\section{Basics.}

If $\Gamma_{0}$ is a subgroup of a discrete group $\Gamma$, then $\Gamma_{0}$ acts on the left on the coset space $\Gamma / \Gamma_{0}$. We say that $\Gamma_{0}$ is almost normal in $\Gamma$, or that $\left(\Gamma, \Gamma_{0}\right)$ 
form an almost normal subgroup pair, if the $\Gamma_{0}$-orbits, $\Gamma_{0}[\gamma]$, in $\Gamma / \Gamma_{0}$ are finite for all $\gamma \in \Gamma([\mathbf{B C}])$.

Proposition 1.1. If $\Gamma_{0}$ is a subgroup of a discrete group $\Gamma$ then $\Gamma_{0}[\gamma] \cong$ $\Gamma_{0} / \Gamma_{0} \cap \gamma \Gamma_{0} \gamma^{-1},(\gamma \in \Gamma)$.

Proof. For each $\gamma \in \Gamma$ consider the map of $\Gamma_{0}$ onto the $\Gamma_{0}$-orbit of $[\gamma]$ defined by $h \mapsto[h \gamma]$. Since $\left\{h \in \Gamma_{0} \mid[h \gamma]=[\gamma]\right\}=\left\{h \in \Gamma_{0} \mid h \gamma \in \gamma \Gamma_{0}\right\}=\Gamma_{0} \cap$ $\gamma \Gamma_{0} \gamma^{-1}$, this map defines a bijection of the coset space $\Gamma_{0} / \Gamma_{0} \cap \gamma \Gamma_{0} \gamma^{-1}$ with $\Gamma_{0}[\gamma]$.

Another set bijection is useful to note. Left multiplication by an element $\alpha$ of $\Gamma$ yields a bijection of $\Gamma / \Gamma_{0}$ with itself, so a subset $M$ of $\Gamma / \Gamma_{0}$ is bijective with $\alpha M$. Setting $M$ to be the orbit $\Gamma_{0}[\gamma]$ and $\alpha$ to be $\gamma^{-1}$, we have that $\Gamma_{0} \gamma \Gamma_{0} / \Gamma_{0} \cong \gamma^{-1} \Gamma_{0} \gamma \Gamma_{0} / \Gamma_{0}$.

For a given almost normal subgroup pair $\left(\Gamma, \Gamma_{0}\right)$, there are $\Gamma_{0}$-bivariant maps $L$ and $R$ from $\Gamma$ to $\mathbb{N}^{\times}$defined by $L(\gamma)=\left|\Gamma_{0}[\gamma]\right|$ and $R(\gamma)=L\left(\gamma^{-1}\right)$ $([\mathbf{B C}])$. The last proposition shows that $L(\gamma)=\left(\Gamma_{0}: \Gamma_{0} \cap \gamma \Gamma_{0} \gamma^{-1}\right)$ and that $[\gamma]$ is a fixed point under the left $\Gamma_{0}$ action, i.e., $L(\gamma)=1$, if and only if $\Gamma_{0} \subseteq \gamma \Gamma_{0} \gamma^{-1}$.

Definition. For an almost normal subgroup pair $\left(\Gamma, \Gamma_{0}\right)$ let $\mathcal{T}=\{\gamma \in \Gamma \mid$ $L(\gamma)=1\}=\left\{\gamma \in \Gamma \mid \Gamma_{0} \gamma \subseteq \gamma \Gamma_{0}\right\}$.

Since $\Gamma_{0} \gamma \subseteq \gamma \Gamma_{0}$ implies $\gamma \Gamma_{0} \subseteq \Gamma_{0} \gamma \Gamma_{0} \subseteq \gamma \Gamma_{0} \Gamma_{0}=\gamma \Gamma_{0}$, and since $\Gamma_{0} \gamma \subseteq$ $\Gamma_{0} \gamma \Gamma_{0}$ it follows that $\mathcal{T}=\left\{\gamma \in \Gamma \mid \Gamma_{0} \gamma \Gamma_{0}=\gamma \Gamma_{0}\right\}$.

Proposition 1.2. If $\left(\Gamma, \Gamma_{0}\right)$ is an almost normal subgroup pair then $\mathcal{T}$ is a subsemigroup of $\Gamma$ and $\mathcal{T} \cap \mathcal{T}^{-1}=N_{\Gamma_{0}}$, the normalizer of $\Gamma_{0}$ in $\gamma$. The map $R: \mathcal{T} \rightarrow \mathbb{N}^{\times}$is a semigroup homomorphism.

Proof. Clearly $e \in \mathcal{T}$. For $\alpha, \beta \in \mathcal{T}$ we have $\Gamma_{0} \subseteq \alpha \Gamma_{0} \alpha^{-1}$ and $\Gamma_{0} \subseteq$ $\beta \Gamma_{0} \beta^{-1}$. Applying the automorphism ad $(\alpha)$ to the second inclusion shows that $\alpha \Gamma_{0} \alpha^{-1} \subseteq \alpha \beta \Gamma_{0} \beta^{-1} \alpha^{-1}$, so $\Gamma_{0} \subseteq(\alpha \beta) \Gamma_{0}(\alpha \beta)^{-1}$ and $\alpha \beta \in \mathcal{T}$. Since $\mathcal{T}^{-1}=\{\gamma \mid R(\gamma)=1\}=\left\{\gamma \mid \gamma \Gamma_{0} \gamma^{-1} \subseteq \Gamma_{0}\right\}$, the second claim is clear. The last assertion follows from the elementary fact that $(G: K)=(G$ : $H)(H: K)$ for subgroups $K \leq H \leq G$ of a group $G$. For $\alpha \beta \in \mathcal{T}$, we have $R(\alpha \beta)=\left(\Gamma_{0}: \Gamma_{0} \cap(\alpha \beta)^{-1} \Gamma_{0} \alpha \beta\right)$ which is equal to $\left(\Gamma_{0}:(\alpha \beta)^{-1} \Gamma_{0} \alpha \beta\right)$ since $\alpha \beta \in \mathcal{T}$. This equals $\left(\Gamma_{0}: \beta^{-1} \Gamma_{0} \beta\right)\left(\beta^{-1} \Gamma_{0} \beta: \beta^{-1} \alpha^{-1} \Gamma_{0} \alpha \beta\right)=\left(\Gamma_{0}:\right.$ $\left.\beta^{-1} \Gamma_{0} \beta\right)\left(\Gamma_{0}: \alpha^{-1} \Gamma_{0} \alpha\right)=R(\beta) R(\alpha)$.

Proposition 1.3. The semigroup homomorphism $R: \mathcal{T} \rightarrow \mathbb{N}^{\times}$defines a map $r: \mathcal{T} / N_{\Gamma_{0}} \rightarrow \mathbb{N}^{\times}$satisfying $r([\alpha \beta])=r([\alpha]) r([\beta])$ for $\alpha, \beta \in \mathcal{T}$.

Proof. For $\alpha \in \mathcal{T}$ set $r([\alpha])=R(\alpha)$. For $\alpha, \beta \in \mathcal{T}$ with $[\alpha]=[\beta]$ we have $\beta^{-1} \alpha \in N_{\Gamma_{0}}=\mathcal{T} \cap \mathcal{T}^{-1}$. Thus $R(\alpha)=R\left(\beta \beta^{-1} \alpha\right)=R(\beta) R\left(\beta^{-1} \alpha\right)=R(\beta)$, the later equality a consequence of $\beta^{-1} \alpha \in \mathcal{T}^{-1}$. Thus $r$ is a well defined map of sets. 
Notice that $\mathcal{T} / N_{\Gamma_{0}}$ is in general only a coset space, so multiplication of elements is not well defined.

We now recall from $[\mathbf{B C}]$ that the Hecke algebra $H\left(\Gamma, \Gamma_{0}\right)$ associated to an almost normal subgroup pair $\left(\Gamma, \Gamma_{0}\right)$ is the convolution algebra of $(\mathbb{C}$ valued say) functions with finite support on $\Gamma_{0} \backslash \Gamma / \Gamma_{0}$, the space of $\Gamma_{0}$-orbits in $\Gamma / \Gamma_{0}$. For $f, h \in H\left(\Gamma, \Gamma_{0}\right)$ define

$$
\begin{aligned}
f * h(\gamma) & =\sum\left\{f(\alpha) h\left(\alpha^{-1} \gamma\right) \mid \alpha \in \Gamma / \Gamma_{0}\right\} \\
f^{*}(\gamma) & =\overline{f\left(\gamma^{-1}\right),} \quad(\gamma \in \Gamma) .
\end{aligned}
$$

Here we view $f$ and $h$ as $\Gamma_{0}$-bivariant functions on $\Gamma$. We now proceed to define some elements of $H\left(\Gamma, \Gamma_{0}\right)$ that will play a basic role in the rest of the paper. For a finite subset $A$ of $\Gamma_{0} \backslash \Gamma / \Gamma_{0}$ let $\chi_{A}$ denote the characteristic function of the set $A$. Also, for $\gamma \in \Gamma$, let $O_{\gamma}$ be the point $\Gamma_{0}[\gamma]=\Gamma_{0} \gamma \Gamma_{0}$ in $\Gamma_{0} \backslash \Gamma / \Gamma_{0}$.

Definition. If $\left(\Gamma, \Gamma_{0}\right)$ is an almost normal subgroup pair and $\gamma \in \mathcal{T}$ let $W_{\gamma}$ be the element of $H\left(\Gamma, \Gamma_{0}\right)$ defined by

$$
W_{\gamma}=R(\gamma)^{-1 / 2} \chi_{O_{\gamma}} .
$$

It will be useful to distinguish those elements $W_{\gamma}$ with $\gamma \in N_{\Gamma_{0}}$ from the others. Write $U_{\gamma}=W_{\gamma}$ if $\gamma \in N_{\Gamma_{0}}$, so $U_{\gamma}=\chi_{O_{\gamma}}$. Note that $O_{\gamma}=\gamma \Gamma_{0}$ for $\gamma \in \mathcal{T}$, so if $\gamma, \gamma^{\prime} \in \mathcal{T}$ then $O_{\gamma}=O_{\gamma^{\prime}}$ if and only if $[\gamma]=\left[\gamma^{\prime}\right]$ in $\mathcal{T} / \Gamma_{0}$. Since $R(\gamma)=R\left(\gamma^{\prime}\right)$ if $[\gamma]=\left[\gamma^{\prime}\right]$ in $\mathcal{T} / \Gamma_{0}$, we have that $W_{\gamma}=W_{\gamma^{\prime}}$ if and only if $[\gamma]=\left[\gamma^{\prime}\right]$ in $\mathcal{T} / \Gamma_{0}$.

Theorem 1.4. For $\left(\Gamma, \Gamma_{0}\right)$ an almost normal subgroup pair, $W: \mathcal{T} \rightarrow$ $H\left(\Gamma, \Gamma_{0}\right)$ is a representation of the semigroup $\mathcal{T}$ by isometries.

Proof. We show first that $W_{\gamma}^{*} W_{\gamma}=W_{e}=I$. For $\beta \in \Gamma, W_{\gamma}^{*} W_{\gamma}(\beta)=$ $R(\gamma)^{-1} \sum\left\{\chi_{O_{\gamma}}\left(\alpha^{-1}\right) \chi_{O_{\gamma}}\left(\alpha^{-1} \beta\right) \mid \alpha \in \Gamma / \Gamma_{0}\right\}$. Since $O_{\gamma}=\gamma \Gamma_{0}$ for $\gamma \in \mathcal{T}$, $\alpha^{-1} \in O_{\gamma}$ if and only if $\alpha \in \Gamma_{0} \gamma^{-1}=\Gamma_{0} \gamma^{-1} \Gamma_{0}$, a set with $L\left(\gamma^{-1}\right)=R(\gamma)$ points in $\Gamma / \Gamma_{0}$. Thus $W_{\gamma}^{*} W_{\gamma}(\beta)=R(\gamma)^{-1} \sum\left\{\chi_{O_{e}}(\beta) \mid \alpha \in \Gamma_{0} \gamma^{-1} \Gamma_{0}\right\}=$ $\chi_{O_{e}}(\beta)=W_{e}(\beta)$. It is clear that $W_{e}$ is the identity of $H\left(\Gamma, \Gamma_{0}\right)$.

For $\alpha, \beta \in \mathcal{T}$ and $\gamma \in \Gamma$ we have

$$
\begin{aligned}
W_{\alpha} W_{\beta}(\gamma) & =(R(\alpha) R(\beta))^{-1 / 2} \sum\left\{\chi_{O_{\alpha}}(\rho) \chi_{O_{\beta}}\left(\rho^{-1} \gamma\right) \mid \rho \in \Gamma / \Gamma_{0}\right\} \\
& =R(\alpha \beta)^{-1 / 2} \chi_{O_{\beta}}\left(\alpha^{-1} \gamma\right),
\end{aligned}
$$

since $O_{\alpha}=\alpha \Gamma_{0}$ is a single point of $\Gamma / \Gamma_{0}$. This expression equals $R(\alpha \beta)^{-1 / 2}$ if and only if $\gamma \in \alpha \Gamma_{0} \beta \Gamma_{0}=\alpha \beta \Gamma_{0}=O_{\alpha \beta}$, and is zero otherwise. Thus $W_{\alpha} W_{\beta}=W_{\alpha \beta}$.

If $\gamma \in N_{\Gamma_{0}}$, we have that $\gamma^{-1} \in N_{\Gamma_{0}}$ also, so $W_{\gamma} W_{\gamma^{-1}}=W_{e}=I$. Thus $W_{\gamma} W_{\gamma}^{*}=W_{\gamma} W_{\gamma}^{*}\left(W_{\gamma} W_{\gamma^{-1}}\right)=W_{\gamma} W_{\gamma^{-1}}=I$ and $U=\left.W\right|_{N_{\Gamma_{0}}}$ is a unitary 
representation of the group $N_{\Gamma_{0}}$ in $H\left(\Gamma, \Gamma_{0}\right)$. Since $\left.W\right|_{\Gamma_{0}}=I, U$ should actually be viewed as a unitary representation of the group $N_{\Gamma_{0}} / \Gamma_{0}$. It follows from $W_{\gamma} W_{\gamma}^{*}=I\left(\gamma \in N_{\Gamma_{0}}\right)$ that for $\alpha \in \mathcal{T}$ the selfadjoint idempotent $W_{\alpha} W_{\alpha}^{*}$ depends only on the equivalence class $[\alpha]$ of $\alpha$ in $\mathcal{T} / N_{\Gamma_{0}}$. Where convenient we denote $W_{\alpha} W_{\alpha}^{*}$ by $P[\alpha]$.

Proposition 1.5. Let $\left(\Gamma, \Gamma_{0}\right)$ be an almost normal subgroup pair, and choose $\alpha \in \mathcal{T}, g \in N_{\Gamma_{0}}$. Then

$$
W_{\alpha} U_{g} W_{\alpha}^{*}=R(\alpha)^{-1} \sum\left\{\chi_{O_{\beta}} \mid \operatorname{ad}\left(\alpha^{-1}\right)(\beta) \in g \Gamma_{0}\right\}
$$

where this sum is over a set of $R(\alpha)$ points in $\Gamma_{0} \backslash \Gamma / \Gamma_{0}$.

Proof. First note that $W_{\alpha} U_{g} W_{\alpha}^{*}=W_{\alpha} W_{g} W_{\alpha}^{*}=W_{\alpha g} W_{\alpha}^{*}$. Since $\alpha g \in \mathcal{T}$, the orbit $O_{\alpha g}=\Gamma_{0} \alpha g \Gamma_{0}$ is the single point $\alpha g \Gamma_{0}$ in $\Gamma / \Gamma_{0}$, so the sum defining the product of $W_{\alpha g}$ with $W_{\alpha}^{*}$ consists only of one non-zero term. We have $W_{\alpha g} W_{\alpha}^{*}(\gamma)=R(\alpha)^{-1} \chi_{O_{\alpha}}\left(\rho^{-1} \gamma\right)^{-1}$ where $\rho=\alpha g \Gamma_{0}$. Since $\rho^{-1} \gamma \in \Gamma_{0} \alpha^{-1}$ if and only if $\gamma \in \alpha g \Gamma_{0} \alpha^{-1}$, this expression is $R(\alpha)^{-1}$ if $\gamma \in \alpha g \Gamma_{0} \alpha^{-1}$ and zero otherwise. Since the set $\Gamma_{0} \alpha^{-1}=\Gamma_{0} \alpha^{-1} \Gamma_{0}$ consists of $L\left(\alpha^{-1}\right)=R(\alpha)$ points in $\Gamma / \Gamma_{0}$, so does the set $\alpha g \Gamma_{0} \alpha^{-1} \Gamma_{0}$. Each one of these points in $\Gamma / \Gamma_{0}$ is however also a point in $\Gamma_{0} \backslash \Gamma / \Gamma_{0}$, since, as already noted, $\Gamma_{0} \alpha g \Gamma_{0}=\alpha g \Gamma_{0}$. Thus $W_{\alpha} U_{g} W_{\alpha}^{*}=R(\alpha)^{-1} \sum\left\{\chi_{O \beta} \mid \beta \in \operatorname{ad}(\alpha)\left(g \Gamma_{0}\right)\right\}$, where the sum has $R(\alpha)$ terms.

Remark 1.6. If we further stipulate that $N_{\Gamma_{0}}$ be normal in $\Gamma$, then the solutions $\beta \in$ ad $(\alpha)\left(g \Gamma_{0}\right)$ occurring in the sum all occur in $N_{\Gamma_{0}}$, in fact, in $N_{\Gamma_{0}} / \Gamma_{0}$, and $\chi_{O_{\beta}}=U_{\beta}$. Thus $W_{\alpha} U_{g} W_{\alpha}^{*}=R(\alpha)^{-1} \sum\left\{U_{\beta} \mid \operatorname{ad}\left(\alpha^{-1}\right)[\beta]=[g]\right.$ in $\left.N_{\Gamma_{0}} / \Gamma_{0}\right\}$.

This remark suggests that if $N_{\Gamma_{0}}$ is normal in $\Gamma$, a certain semigroup $C^{*}$-dynamical system associated to an almost normal subgroup pair $\left(\Gamma, \Gamma_{0}\right)$ should be considered. More generally, if $N$ is a normal subgroup of $\Gamma$ with $\Gamma_{0}<N<N_{\Gamma_{0}}$, then ad $\left(\alpha^{-1}\right) N=N$ for $\alpha \in \Gamma$ and since ad $\left(\alpha^{-1}\right) \Gamma_{0} \subseteq \Gamma_{0}$ for any $\alpha \in \mathcal{T}$, the map ad $\left(\alpha^{-1}\right)$ defines a group homomorphism of $N / \Gamma_{0}$ to itself, for any $\alpha \in \mathcal{T}$. Thus, if $g \in N$, we may replace $N_{\Gamma_{0}}$ by $N$ in the above remark. Define an action $\Theta$ of the semigroup $\mathcal{T}$ on $l^{1}\left(N / \Gamma_{0}\right)$ by $\Theta_{\alpha}(f)=R(\alpha)^{-1} \cdot f \circ$ ad $\left(\alpha^{-1}\right)$, for $\alpha \in \mathcal{T}, f \in l^{1}\left(N / \Gamma_{0}\right)$. For $g \in N / \Gamma_{0}$ let $\delta_{g}$ be the element of $l^{1}\left(N / \Gamma_{0}\right)$ which is one at $g$ and zero elsewhere. Compute that

$$
\Theta_{\alpha}\left(\delta_{g}\right)=R(\alpha)^{-1} \sum\left\{\delta_{\beta} \mid \operatorname{ad}\left(\alpha^{-1}\right)(\beta)=g\right\}
$$

so $\left\|\Theta_{\alpha}\left(\delta_{g}\right)\right\|_{1} \leq\left\|\delta_{g}\right\|_{1}=1$. Thus $\Theta$ is a continuous action of the semigroup $\mathcal{T}$ on the Banach $*$-algebra $l^{1}\left(N / \Gamma_{0}\right)$, so defines an action, again denoted by $\Theta$, of $\mathcal{T}$ on the $C^{*}$-completion, $C^{*}\left(N / \Gamma_{0}\right)$. Thus, to any almost normal subgroup pair $\left(\Gamma, \Gamma_{0}\right)$ and $N$ a normal subgroup of $\Gamma$ containing $\Gamma_{0}$, and 
contained in the normalizer of $\Gamma_{0}$, there is a semigroup $C^{*}$-dynamical system $\left(C^{*}\left(N / \Gamma_{0}\right), \Theta, \mathcal{T}\right)$

For the reader's convenience we recall some facts concerning semigroup dynamical systems. For further details see the results in $[\mathbf{L R}]$ and the references therein. A covariant representation of a semigroup $C^{*}$-dynamical system $(\mathcal{A}, \Theta, S)$ where $\Theta$ is a representation of the semigroup $S$ as (possibly non-unital) endomorphisms of a unital $C^{*}$-algebra $\mathcal{A}$, is a pair $(\pi, V)$ with $\pi$ a unital representation of $\mathcal{A}$ on a Hilbert space $\mathcal{H}$ and $V$ a representation of $S$ on $\mathcal{H}$ as isometries with $V_{e}=I$, such that $\pi\left(\Theta_{s}(a)\right)=V_{s} \pi(a) V_{s}^{*}$, $a \in \mathcal{A}$ and $s \in S$. If a semigroup dynamical system $(\mathcal{A}, \Theta, S)$ possesses a covariant representation $(\pi, V)$ then there is a unique unital $C^{*}$-algebra $\mathcal{A} \rtimes S$ equipped with a unital homomorphism $i: \mathcal{A} \rightarrow \mathcal{A} \rtimes S$ and a representation $\nu: S \rightarrow \mathcal{A} \rtimes S$ by isometries so that $(i, \nu)$ satisfies the covariance relation, their images generate $\mathcal{A} \rtimes S$ as a $C^{*}$-algebra, and such that every covariant representation $(\rho, W)$ of $(\mathcal{A}, \Theta, S)$ yields a representation $\rho \times W$ of $\mathcal{A} \rtimes S$ with $(\rho \times W) \circ i=\rho$ and $(\rho \times W) \circ \nu=W$.

We return now to our context, namely an almost normal subgroup pair $\left(\Gamma, \Gamma_{0}\right)$ and a normal subgroup $N$ of $\Gamma$ with $\Gamma_{0}<N<N_{\Gamma_{0}}$. If $\pi$ : $H\left(\Gamma, \Gamma_{0}\right) \rightarrow \mathcal{B}(\mathcal{H})$ is a $*$-representation of the Hecke algebra $H\left(\Gamma, \Gamma_{0}\right)$ as bounded operators on a Hilbert space $\mathcal{H}$, denote by $\pi_{W}$ the representation of $\mathcal{T}$ as partial isometries on $\mathcal{H}$ given by $\pi_{W}=\pi \circ W$, and denote by $\pi_{U}$ the unitary representation of the group $N / \Gamma_{0}$ on $\mathcal{H}$ given by $\pi_{U}=\pi \circ U$. Combining Proposition 1.5 and Remark 1.6 with the above expression for $\Theta_{\alpha}\left(\delta_{g}\right), \alpha \in \mathcal{T}$ and $g \in N / \Gamma_{0}$, gives the following result.

Theorem 1.7. If $\left(\Gamma, \Gamma_{0}\right)$ is an almost normal subgroup pair and $N$ a normal subgroup of $\Gamma$ with $\Gamma_{0}<N<N_{\Gamma_{0}}$ then to every $*$-representation $\pi$ : $H\left(\Gamma, \Gamma_{0}\right) \rightarrow B(\mathcal{H})$ there corresponds a covariant representation $\left(\pi_{U}, \pi_{W}\right)$ of the semigroup $C^{*}$-dynamical system $\left(C^{*}\left(N / \Gamma_{0}\right), \Theta, \mathcal{T}\right)$ on $\mathcal{H}$.

Corollary 1.8. The $C^{*}$-algebra $C^{*}\left(N / \Gamma_{0}\right) \rtimes \mathcal{T}$ exists and for each $*$-representation $\pi$ of $H\left(\Gamma, \Gamma_{0}\right)$ there is a $*$-representation $\rho$ of $C^{*}\left(N / \Gamma_{0}\right) \rtimes \mathcal{T}$ with image contained in the $C^{*}$-algebra generated by $\operatorname{Im}(\pi)$ and with $\rho \circ i=\pi_{U}$ and $\rho \circ \nu=\pi_{W}$.

Proof. To conclude that the $C^{*}$-semicrossed product algebra exists it is enough to show that there is at least one covariant representation of the dynamical system $\left(C^{*}\left(N / \Gamma_{0}\right), \Theta, \mathcal{T}\right)$. This follows from Theorem 1.7 after noting that there is always a regular representation of $H\left(\Gamma, \Gamma_{0}\right)$ on the Hilbert space $l^{2}\left(\Gamma / \Gamma_{0}\right)([\mathbf{B C}])$.

Under certain conditions, we may consider a slightly less cumbersome semigroup $C^{*}$-dynamical system. Consider the situation of a normal subgroup $N$ of a discrete group $\Gamma$. There is a commuting diagram of groups 
with exact rows:

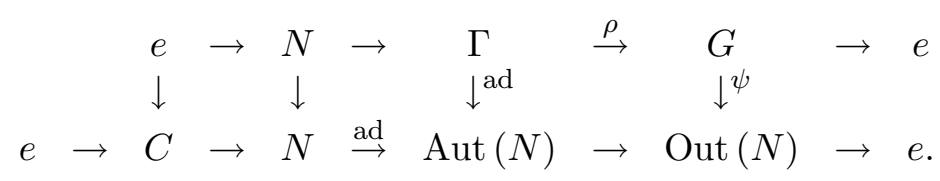

Here $C$ is the center of $N, G=\Gamma / N$, ad $: \Gamma \rightarrow \operatorname{Aut}(N)$ is defined by $\left.\gamma \rightarrow \operatorname{ad}(\gamma)\right|_{N}$, and $\psi$ is defined by the diagram. If $\Gamma_{0}$ is any normal subgroup of $N$, then ad $(\gamma)\left(\Gamma_{0}\right)=\Gamma_{0}$ for any $\gamma \in N$, so for $g \in G$ we may denote by $\psi_{g}\left(\Gamma_{0}\right)$ the well defined subgroup ad $(\gamma)\left(\Gamma_{0}\right)$ where $\gamma \in \Gamma$ is any element of $\rho^{-1}(g)$.

Lemma 1.9. Given the above diagram and $\Gamma_{0}$ a normal subgroup of $N$, the subgroup pair $\left(\Gamma, \Gamma_{0}\right)$ is almost normal if and only if the subgroup $\psi_{g}\left(\Gamma_{0}\right) \Gamma_{0} / \Gamma_{0}$ of $N / \Gamma_{0}$ is finite for each $g \in G$.

Proof. The subgroup $\gamma \Gamma_{0} \gamma^{-1} \Gamma_{0} / \Gamma_{0}=\operatorname{ad}(\gamma)\left(\Gamma_{0}\right) \Gamma_{0} / \Gamma_{0}$ is finite for each $\gamma \in$ $\Gamma$ if and only if $\psi_{g}\left(\Gamma_{0}\right) \Gamma_{0} / \Gamma_{0}$ is finite for each $g \in G$. The comment after Proposition 1.1 finishes the claim.

Assume now that $\left(\Gamma, \Gamma_{0}\right)$ is an almost normal subgroup pair. Then $\rho(\mathcal{T})=$ $\mathcal{T} / N=\left\{g \in G \mid \Gamma_{0} \subseteq \psi_{g}\left(\Gamma_{0}\right)\right\}$ is a semigroup of $G$, denote it by $T$. The normalizer of $\Gamma_{0}$ is equal to $N$ if and only if $\left\{g \in G \mid \psi_{g}\left(\Gamma_{0}\right)=\Gamma_{0}\right\}$, which is $T \cap T^{-1}$, is equal to $e$. Otherwise $N_{\Gamma_{0}}$ is an extension of $N$ by $T \cap T^{-1}$. Proposition 1.3 shows that the map $R: \mathcal{T} \rightarrow \mathbb{N}^{\times}$defines a semigroup homomorphism of $\mathcal{T} / N$ to $\mathbb{N}^{\times}$. Also, for $\alpha \in N$, the group endomorphism ad $\left(\alpha^{-1}\right)$ of $N / \Gamma_{0}$ is actually an automorphism of $N / \Gamma_{0}$. If there is a splitting homomorphism $\nu: G \rightarrow \Gamma$, in other words if $\Gamma$ is the semidirect product of $N$ by $G$, then $\nu(g) \in \mathcal{T}$ for $g \in T$, so we may define a semigroup $C^{*}$-dynamical system $\left(C^{*}\left(N / \Gamma_{0}\right), \tilde{\Theta}, T\right)$ by setting $\tilde{\Theta}=\Theta \circ \nu$. If $\pi$ is a $*$-representation of $H\left(\Gamma, \Gamma_{0}\right)$ in $\mathcal{B}(\mathcal{H})$ then set $\pi_{T}=\pi \circ W \circ \nu$ and, as before, set $\pi_{U}=\pi \circ U$. The pair $\left(\pi_{U}, \pi_{T}\right)$ is a covariant representation of this dynamical system. Summarizing these observations in connection with the previous results gives the following result.

Theorem 1.10. If $\left(\Gamma, \Gamma_{0}\right)$ is an almost normal subgroup pair and $e \rightarrow$ $N \rightarrow \Gamma \stackrel{\rho}{\rightarrow} G \rightarrow e$ is a split exact sequence with $\Gamma_{0} \unlhd N$ then to every *-representation $\pi: H\left(\Gamma, \Gamma_{0}\right) \rightarrow \mathcal{B}(\mathcal{H})$ there corresponds a covariant representation $\left(\pi_{T}, \pi_{U}\right)$ of the semigroup $C^{*}$-dynamical system $\left(C^{*}\left(N / \Gamma_{0}\right), \tilde{\Theta}, T\right)$ on $\mathcal{H}$. The $C^{*}$-algebra $C^{*}\left(N / \Gamma_{0}\right) \rtimes T$ exists, and given $\pi$ there is a*representation $\pi^{\prime}$ of $C^{*}\left(N / \Gamma_{0}\right) \rtimes T$ with image contained in the $C^{*}$-algebra generated by $\operatorname{Im}(\pi)$, and with $\pi^{\prime} \circ i=\pi_{U}, \pi^{\prime} \circ \nu=\pi_{T}$.

For example, consider the case when $\Gamma_{0}$ is a normal subgroup of $\Gamma$. It is clear that the Hecke algebra $H\left(\Gamma, \Gamma_{0}\right)$ is the group algebra $\mathbb{C}\left[\Gamma / \Gamma_{0}\right]$. Setting $N=N_{\Gamma_{0}}$, so $N=\Gamma$, we have $G=\{e\}$ and Theorem 1.10 gives a 
representation $\pi^{\prime}$ of $C^{*}\left(\Gamma / \Gamma_{0}\right)$ in the $C^{*}$-algebra generated by $\operatorname{Im}(\pi)$ for any representation $\pi$ of $\Gamma / \Gamma_{0}$. Of course, in this context, Theorem 1.10 is far from the best possible.

\section{Order Structure.}

The semigroup $\mathcal{T}$ of $\Gamma$ defines a pre-order type structure on $\Gamma$. Some of the extra structure on this ordering that is of consequence for our context has been developed before in $[\mathbf{N}]$. We develop some of the slightly more general results that are needed in our situation though.

Let $S$ be a sub-semigroup of a discrete group $G$ and define a relation $\precsim$ on $G$ by $a \precsim b$ if and only if $a^{-1} b \in S$. This relation is: Reflexive since $e \in S$; transitive since $S S \subseteq S$; and left invariant, i.e., $a \precsim b$ implies $g a \precsim g b$ for $g \in G$. We do not specify that the subgroup $S \cap S^{-1}=\{e\}$, so the relation is not a partial order on $G$. The elements $a, b$ satisfy both $a \precsim b$ and $b \precsim a$ if and only if $a^{-1} b \in S \cap S^{-1}$. As noted in $[\mathbf{N}]$, the set $S S^{-1}=\{g \in G \mid \widetilde{g}$ has an upper bound in $S\}$. If any two arbitrarily chosen elements of a subset $A$ in $G$ have a common upper bound (c.u.b) in $G$ call $A$ upward directed.

Lemma 2.1. A subset $A$ of $G$ is upward directed if and only if $A^{-1} A \subseteq$ $S S^{-1}$.

Proof. First suppose that $A$ is upward directed. If $l$ is a c.u.b. for a pair $a, b$ in $A$, then $a^{-1} b=a^{-1} l\left(b^{-1} l\right)^{-1} \in S S^{-1}$. For the reverse implication first notice that if $h=s t^{-1}$ with $s, t \in S$ then $h$ and $e$ have a c.u.b., namely $s$. Given $a, b \in A$ arbitrary, $b^{-1} a \in S S^{-1}$ by hypothesis, so $b^{-1} a$ and $e$ have a c.u.b., say $l$. By left invariance, $a \precsim b l$ and $b \precsim b l$, so $b l$ is a c.u.b. for the pair $a, b$ of $A$.

Thus $S$ is upward directed if and only if $S^{-1} S \subseteq S S^{-1}$ (cf [N, $\left.2.2 .2\right]$ ). Also $G$ is upward directed if and only if $G=S S^{-1}$.

For $a, b \in G$, denote by $a \vee b$ a least upper bound for $a$ and $b$, if it exists. The set of least upper bounds for $a$ and $b$ is $a \vee b\left(S \cap S^{-1}\right)$. The following fact is a straightforward consequence of left invariance for the relation $\precsim$.

Lemma 2.2. Given a pair $a, b$ in $G$ so that $a \vee b$ exists, and $g \in G$, then $g(a \vee b)$ is a l.u.b. for the pair $g a, g b$.

With an analogous definition for a subset $A$ of $G$ to be lower directed, it follows that $A$ is lower directed if and only if $A^{-1} A \subseteq S^{-1} S$. For example, if $l$ is a common lower bound (c.l.b) for $a, b$ in $A$ then $a^{-1} b=\left(l^{-1} a\right)^{-1}\left(l^{-1} b\right) \in$ $S^{-1} S$. In the other direction, if $h=s^{-1} t \in S^{-1} S$ then $s^{-1}$ is a c.l.b. for $h, e$. Thus, for $a, b \in A, a^{-1} b \in S^{-1} S$ so there is a c.l.b., say $l$, for $a^{-1} b, e$. Then $a l$ is a c.l.b. for $a, b$.

Thus $S^{-1}$ is lower directed if and only if $S S^{-1} \subseteq S^{-1} S$ and $G$ is lower directed if and only if $G=S^{-1} S$. Note also that if $A \subseteq G$ is lower directed 
and if $a \vee b$ exists for $a, b \in S$ then $g \vee h$ exists for all $g, h \in A$. To see this, let $c$ be a c.l.b. for $g$ and $h$. Then $c^{-1} g, c^{-1} h \in S$, so $c^{-1} g \vee c^{-1} h$ exists. Then $c\left(c^{-1} g \vee c^{-1} h\right)$ is a l.u.b. for $g$ and $h$.

Theorem 2.3. Assume any pair of elements in $S$ have a l.u.b. If $S S^{-1} \subseteq$ $S^{-1} S$ then $e \vee g$ exists for any $g \in S S^{-1}$. In particular if $S \cap S^{-1}=\{e\}$, then $(G, S)$ is a quasi-lattice ordered group.

Proof. Since $S S^{-1} \subseteq S^{-1} S, S^{-1}$ is lower directed. By the remarks preceding the theorem, $c^{-1} \vee d^{-1}$ exists for any $c, d \in S$. Thus $c\left(c^{-1} \vee d^{-1}\right)$ is a l.u.b. for $e$ and $c d^{-1}$. Set $g=c d^{-1}$.

We may, following $[\mathbf{N}]$, define a partially pre-ordered group $(G, S)$ to be quasi-lattice pre-ordered if

1) $e \vee g$ exists for $g \in S S^{-1}$.

2) For $a, b \in S$ with a c.u.b, $a \vee b$ exists.

As an example, consider the following: $G=\{g \in G L(d, \mathbb{Q}) \mid \operatorname{det} g>$ $0\}$ and $S=\left\{g \in \mathfrak{M}_{d}(\mathbb{Z}) \mid \operatorname{det} g \geq 1\right\}$. Then $S \cap S^{-1}=S L(d, \mathbb{Z})$ and $G=S S^{-1}=S^{-1} S$. In fact $G=\mathbb{N}^{-1} S$ where $n \in \mathbb{N}$ is identified with the diagonal matrix $n \otimes I_{d}$ and $S=\left\{g \in G \mid g\left(\mathbb{Z}^{d}\right) \subseteq \mathbb{Z}^{d}\right\}$. Thus, for $a, b \in G$, we have $a \precsim b$ if and only if $b\left(\mathbb{Z}^{d}\right) \subseteq a\left(\mathbb{Z}^{d}\right)$. It is known that $a \vee b$ exists for any $a, b \in S$, usually known as the least common multiple of $a$ and $b$. Theorem 2.3 shows that $(G, S)$ is a quasi-lattice pre-ordered group.

Remark 2.4. One last observation before we return to our own context. If $(G, S)$ is a pre-ordered group such that 1$)$ above is satisfied, then any $x \in S S^{-1}$ may be written as $s t^{-1}$ for some $s, t \in S$ with $s^{-1} \vee t^{-1}=e$. For example, set $s$ to be a l.u.b. for $e$ and $x$ and set $t=x^{-1} s$. Then $s, t \in S$ and $x=s t^{-1}$. Since $e \vee x=s$, it follows that $s^{-1} \vee t^{-1}=s^{-1} \vee s^{-1} x=$ $s^{-1}(e \vee x)=e$.

Returning to the situation of an almost normal subgroup pair $\left(\Gamma, \Gamma_{0}\right)$ with $\mathcal{T}$ the semigroup of $\Gamma$ defined by $\mathcal{T}=\left\{\alpha \in \Gamma \mid \Gamma_{0} \subseteq\right.$ ad $\left.(\alpha) \Gamma_{0}\right\}$ it follows that $\alpha \precsim \beta$ in $\Gamma$ with the pre-order defined by $\mathcal{T}$ if and only if $\alpha \Gamma_{0} \alpha^{-1} \subseteq \beta \Gamma_{0} \beta^{-1}$.

Lemma 2.5. Let $\alpha, \beta \in \Gamma$ where $\left(\Gamma, \Gamma_{0}\right)$ is an almost normal subgroup pair. Assume there is a $\gamma \in \Gamma$ with ad $(\gamma) \Gamma_{0}=\operatorname{ad}(\alpha) \Gamma_{0}$ ad $(\beta) \Gamma_{0}$. Then $\gamma$ is a l.u.b. for $\alpha$ and $\beta$. If $\eta$ is any l.u.b. for $\alpha$ and $\beta$ then $\operatorname{ad}(\eta) \Gamma_{0}=\operatorname{ad}(\gamma) \Gamma_{0}$. We also have $\operatorname{ad}(\alpha) \Gamma_{0} \operatorname{ad}(\beta) \Gamma_{0}=\operatorname{ad}(\beta) \Gamma_{0} \operatorname{ad}(\alpha) \Gamma_{0}$.

Proof. Any common upper bound $\delta$ for $\alpha$ and $\beta$ satisfies ad $(\alpha) \Gamma_{0} \subseteq \operatorname{ad}(\delta) \Gamma_{0}$ and $\operatorname{ad}(\beta) \Gamma_{0} \subseteq \operatorname{ad}(\delta) \Gamma_{0}$, so ad $(\alpha) \Gamma_{0}$ ad $(\beta) \Gamma_{0} \subseteq \operatorname{ad}(\delta) \Gamma_{0}$. Thus $\gamma$ is clearly a l.u.b. for $\alpha$ and $\beta$. If $\eta$ is another l.u.b. for $\alpha$ and $\beta$, then $\eta^{-1} \gamma \in \mathcal{T} \cap$ $\mathcal{T}^{-1}=N_{\Gamma_{0}}$, so ad $(\gamma) \Gamma_{0}=\operatorname{ad}(\eta) \Gamma_{0}$. Since ad $(\alpha) \Gamma_{0} \operatorname{ad}(\beta) \Gamma_{0}$ is a subgroup $H=\operatorname{ad}(\gamma) \Gamma_{0}$ of $\Gamma, H=H^{-1}=\operatorname{ad}(\beta) \Gamma_{0} \operatorname{ad}(\alpha) \Gamma_{0}$. 
A l.u.b. $\gamma \in \Gamma$ for $\alpha, \beta \in \Gamma$ that satisfies ad $(\alpha) \Gamma_{0}$ ad $(\beta) \Gamma_{0}=\operatorname{ad}(\gamma) \Gamma_{0}$ will be referred to as a solvable l.u.b. We write $\gamma=\alpha \vee_{s} \beta$. The previous results on least upper bounds hold in $(\Gamma, \mathcal{T})$ with l.u.b. replaced by solvable l.u.b. The example described after Theorem 2.3 actually has solvable least upper bounds.

Lemma 2.6. Let $\left(\Gamma, \Gamma_{0}\right)$ be an almost normal subgroup pair. If $\alpha, \beta \in \mathcal{T}$ with $\alpha^{-1} \vee_{s} \beta^{-1}=e$, then $W_{\alpha} W_{\beta}^{*}=(R(\alpha \beta))^{-1 / 2} \chi_{O_{\alpha \beta}-1}$.

Proof. We have $W_{\alpha} W_{\beta}^{*}(\gamma)=(R(\alpha \beta))^{-1 / 2} \chi_{O_{\beta}}\left(\left(\rho^{-1} \gamma\right)^{-1}\right)$ where $\rho=\alpha \Gamma_{0}$. This is zero, except when $\gamma \in \alpha \Gamma_{0} \beta^{-1}=\operatorname{ad}(\alpha) \Gamma_{0} \alpha \beta^{-1}$. Now $O_{\alpha \beta^{-1}}=$ $\Gamma_{0} \alpha \beta^{-1} \Gamma_{0}=\Gamma_{0}$ ad $\left(\alpha \beta^{-1}\right) \Gamma_{0} \alpha \beta^{-1}$. Thus, the equality holds if and only if $\operatorname{ad}(\alpha) \Gamma_{0}=\Gamma_{0}$ ad $\left(\alpha \beta^{-1}\right) \Gamma_{0}$, which is equivalent to $\alpha^{-1} \vee_{s} \beta^{-1}=e$.

We now consider the problem of finding norms on $H\left(\Gamma, \Gamma_{0}\right)$. As in $[\mathbf{B C}]$, there is an $L^{1}$ norm on $H\left(\Gamma, \Gamma_{0}\right)$. For $f$ in $H\left(\Gamma, \Gamma_{0}\right)$ let

$$
\begin{aligned}
\|f\|_{1} & =\sum_{\gamma \in \Gamma_{0} \backslash \Gamma / \Gamma_{0}}(R(\gamma) L(\gamma))^{1 / 2}|f(\gamma)| \\
& =\sum_{\gamma \in \Gamma / \Gamma_{0}} \delta(\gamma)^{-1 / 2}|f(\gamma)|
\end{aligned}
$$

where $\delta(\gamma)=L(\gamma) / R(\gamma)$. In order to define a universal $C^{*}$-norm on $H\left(\Gamma, \Gamma_{0}\right)$ the next lemma will be useful.

Lemma 2.7. If $\alpha, \beta \in \mathcal{T}$ and $\alpha^{-1} \vee_{s} \beta^{-1}=e$ then $L\left(\alpha \beta^{-1}\right)=R(\beta)$ and $R\left(\alpha \beta^{-1}\right)=R(\alpha)$.

Proof. Since $\alpha^{-1} \vee_{s} \beta^{-1}=e$, we have $\alpha \Gamma_{0} \beta^{-1}=\Gamma_{0} \alpha \beta^{-1} \Gamma_{0}$, so $L\left(\alpha \beta^{-1}\right)$ is the number of left cosets in $\Gamma / \Gamma_{0}$ of $\alpha \Gamma_{0} \beta^{-1}=\alpha \Gamma_{0} \beta^{-1} \Gamma_{0}$, which in turn is the number of left cosets in $\Gamma / \Gamma_{0}$ of $\Gamma_{0} \beta^{-1} \Gamma_{0}$. This is of course $L\left(\beta^{-1}\right)=$ $R(\beta)$. Since $\beta^{-1} \vee_{s} \alpha^{-1}=e$ also, it follows that $L\left(\beta \alpha^{-1}\right)=R(\alpha)$. So $R\left(\alpha \beta^{-1}\right)=R(\alpha)$.

If we assume that $\Gamma$ is upward directed, in other words that $\Gamma=\mathcal{T} T^{-1}$, then any $f \in H\left(\Gamma, \Gamma_{0}\right)$ may be written as a finite $\operatorname{sum} \sum\left\{a_{i} \chi_{O_{\alpha_{i} \beta_{i}^{-1}}} \mid \alpha_{i}, \beta_{i} \in\right.$ $\left.\mathcal{T}, a_{i} \in \mathbb{C}\right\}$. If we further assume that $\mathcal{T}^{-1} \mathcal{T}=\Gamma$ and that any pair of elements in $\mathcal{T}$ have a solvable l.u.b., then by Theorem 2.3 and Remark 2.4, we can ensure that any $f \in H\left(\Gamma, \Gamma_{0}\right)$ may be written as a finite $\operatorname{sum} \sum\left\{a_{i} \chi_{O_{\alpha_{i} \beta_{i}^{-1}}} \mid \alpha_{i}, \beta_{i} \in \mathcal{T}, \alpha_{i}^{-1} \vee_{s} \beta^{-1}=e, a_{i} \in \mathbb{C}\right\}$. If $\pi$ is a *-representation of $H\left(\Gamma, \Gamma_{0}\right)$ as bounded operators on a Hilbert space, then, for $f$ of this form, $\pi(f)=\sum a_{i} R\left(\alpha_{i} \beta_{i}\right)^{1 / 2} \pi\left[W_{\alpha_{i}} W_{\beta_{i}}^{*}\right]$ by Lemma 2.6. Thus $\|\pi(f)\| \leq \sum\left|a_{i}\right| R\left(\alpha_{i}\right)^{1 / 2} R\left(\beta_{i}\right)^{1 / 2}=\sum\left|a_{i}\right| R\left(\alpha_{i} \beta_{i}^{-1}\right)^{1 / 2} L\left(\alpha_{i} \beta_{i}^{-1}\right)^{1 / 2}=\|f\|_{1}$, 
the first equality following from Lemma 2.7. Summarizing this gives the next proposition.

Proposition 2.8. Let $\left(\Gamma, \Gamma_{0}\right)$ be an almost normal subgroup pair satisfying $\Gamma=\mathcal{T}^{-1} \mathcal{T}=\mathcal{T} T^{-1}$ and so that any pair of elements of $\mathcal{T}$ have a solvable l.u.b. Then $H\left(\Gamma, \Gamma_{0}\right)=\operatorname{span}_{\mathbb{C}}\left\{W_{\alpha} W_{\beta}^{*} \mid \alpha, \beta \in \mathcal{T}, \alpha^{-1} \vee_{s} \beta^{-1}=e\right\}$. Also $\|f\|=\sup \left\{\|\pi(f)\| \mid \pi: H\left(\Gamma, \Gamma_{0}\right) \rightarrow B(\mathcal{H})\right.$ a $*$-representation $\}$ defines a $C^{*}$ norm on $H\left(\Gamma, \Gamma_{0}\right)$. Denote the $C^{*}$-completion of $H\left(\Gamma, \Gamma_{0}\right)$ in this norm by $C^{*}\left(\Gamma, \Gamma_{0}\right)$.

Remark 2.9. One may define the almost normal subgroup pair $\left(\Gamma, \Gamma_{0}\right)$ to be amenable, under the hypothesis of the Proposition, if \|\|$\leq\|\|_{r}$ on $H\left(\Gamma, \Gamma_{0}\right)$, where \|\|$_{r}$ is the norm on $H\left(\Gamma, \Gamma_{0}\right)$ arising from the regular representation of $H\left(\Gamma, \Gamma_{0}\right)$ on $l^{2}\left(\Gamma / \Gamma_{0}\right)([\mathbf{B C}])$.

The situation discussed previously in Section 1, with $N$ a normal subgroup of $\Gamma$ and $\Gamma_{0} \unlhd N$ leads to further conclusions for $\left(\Gamma, \Gamma_{0}\right)$ an almost normal subgroup pair satisfying the hypothesis of Proposition 2.8. We immediately have the following for example.

Proposition 2.10. If $\left(\Gamma, \Gamma_{0}\right)$ is an almost normal subgroup pair with $\Gamma=$ $\mathcal{T}^{-1} \mathcal{T}=\mathcal{T} T^{-1}$ and such that $\alpha \vee_{s} \beta$ exists for $\alpha, \beta \in \mathcal{T}$, and if $N$ is a normal subgroup of $\Gamma$ with $\Gamma_{0} \leq N \leq N_{\Gamma_{0}}$, then there is a natural surjective *-homomorphism of $C^{*}\left(N / \Gamma_{0}\right) \rtimes \mathcal{T}$ onto $C^{*}\left(\Gamma, \Gamma_{0}\right)$.

Proof. Using the definition of $C^{*}\left(\Gamma, \Gamma_{0}\right)$, Corollary 1.8 gives a $*$-representation $\pi$ of $C^{*}\left(N / \Gamma_{0}\right) \rtimes \mathcal{T}$ into $C^{*}\left(\Gamma, \Gamma_{0}\right)$. Proposition 2.8 shows that the image of $H\left(\Gamma, \Gamma_{0}\right)$ in $C^{*}\left(\Gamma, \Gamma_{0}\right)$ is contained in the image of $\pi$, so $\pi$ is a surjection.

Of course we can say more with the hypothesis of this proposition. With $G=\Gamma / N=\rho(\Gamma)$ and $T=\mathcal{T} / N$ as before, we can carry the order structure of $(\Gamma, \mathcal{T})$ to $(G, T)$. Thus $g \leq h$ in $G$ if and only if $\psi_{g}\left(\Gamma_{0}\right) \subseteq \psi_{h}\left(\Gamma_{0}\right)$, where $\psi$ is defined after Corollary 1.8. In particular, the definition of solvable least upper bound remains compatible, so $\alpha \vee_{s} \beta=\gamma$ in $\Gamma$ if and only if $\rho(\alpha) \vee_{s}$ $\rho(\beta)=\rho(\gamma)$. In the particular case that $N=N_{\Gamma_{0}}$, then $T \cap T^{-1}=\{e\}$, so $(G, T)$ is a partially ordered group (the order relation is antisymmetric) and least upper bounds, if they exist, are unique. Also note that $G=T^{-1} T$ if and only if $\Gamma=\mathcal{T}^{-1} \mathcal{T}$. To see this, it is enough to show that $G=T^{-1} T$ implies $\Gamma=\mathcal{T}^{-1} \mathcal{T}$. Choosing $\gamma \in \Gamma$, we have $\rho(\gamma)=\rho\left(\alpha^{-1} \beta\right)$ some $\alpha, \beta \in \mathcal{T}$. Thus $\gamma \in \alpha^{-1} \beta N$ so $\gamma \in \mathcal{T}^{-1} \mathcal{T}$, since $N \leq N_{\Gamma_{0}} \leq \mathcal{T}$. Similarly, $G=T T^{-1}$ if and only if $\Gamma=\mathcal{T} \mathcal{T}^{-1}$.

Theorem 2.11. If $\left(\Gamma, \Gamma_{0}\right)$ is an almost normal subgroup pair and $e \rightarrow N \rightarrow$ $\Gamma \stackrel{\rho}{\rightarrow} G \rightarrow e$ is a split exact sequence with $\Gamma_{0} \unlhd N$, and if $G=T T^{-1}=T^{-1} T$ so that the solvable least upper bound $g \vee_{s} h$ exists for every pair $g, h \in T$, 
then there is a natural surjective $*$-homomorphism of $C^{*}\left(N / \Gamma_{0}\right) \rtimes T$ onto $C^{*}\left(\Gamma, \Gamma_{0}\right)$.

Proof. Since $G=T T^{-1}=T^{-1} T$, we have $\Gamma=\mathcal{T}^{-1} \mathcal{T}=\mathcal{T} T^{-1}$. Also, the solvable l.u.b. exists for any pair $\alpha, \beta$ in $\mathcal{T}$. Proposition 2.8 shows that $C^{*}\left(\Gamma, \Gamma_{0}\right)$ exists, so Theorem 1.10 yields a $*$-homomorphism $\pi: C^{*}\left(N / \Gamma_{0}\right) \rtimes$ $T$ to $C^{*}\left(\Gamma, \Gamma_{0}\right)$. Proposition 2.8 allows us to see that the image of $H\left(\Gamma, \Gamma_{0}\right)$ in $C^{*}\left(\Gamma, \Gamma_{0}\right)$ is contained in the image of $\pi$, so $\pi$ is a surjection.

\section{Universal Properties.}

In this section our main goal is to provide certain conditions under which the Hecke $C^{*}$-algebra is isomorphic to a semigroup crossed product $C^{*}$-algebra. A crucial role is played here by the covariance condition of Nica, $[\mathbf{N}]$.

Lemma 3.1. If $\alpha, \beta \in \mathcal{T}$ with $\alpha \vee_{s} \beta=\gamma$ then $R(\gamma)\left|\operatorname{ad}(\alpha) \Gamma_{0} \cap \operatorname{ad}(\beta) \Gamma_{0}\right|=$ $R(\alpha) R(\beta)$, where the cardinality is computed in $\Gamma / \Gamma_{0}$.

Proof. For $\alpha \in \mathcal{T}$, the cardinality of ad $(\alpha) \Gamma_{0}=\alpha \Gamma_{0} \alpha^{-1} \Gamma_{0}$ in $\Gamma / \Gamma_{0}$ is the same as the cardinality of $\Gamma_{0} \alpha^{-1} \Gamma_{0}$ in $\Gamma / \Gamma_{0}$, which is $R(\alpha)$. Since ad $(\gamma) \Gamma_{0}=$ $\operatorname{ad}(\alpha) \Gamma_{0} \operatorname{ad}(\beta) \Gamma_{0}, \operatorname{ad}(\gamma) \Gamma_{0}=\bigcup\left\{\eta \operatorname{ad}(\beta) \Gamma_{0} \mid \eta \in \operatorname{ad}(\alpha) \Gamma_{0}\right\}$. The left cosets $\eta \operatorname{ad}(\beta) \Gamma_{0}$ are either disjoint or coincide as $\eta$ varies, and as sets, each is isomorphic to $a d(\beta) \Gamma_{0}$, which has $R(\beta)$ elements in $\Gamma / \Gamma_{0}$. Since two such cosets given by $\eta$ and $\eta^{\prime}$ coincide if and only if $\eta^{\prime-1} \eta \in \operatorname{ad}(\beta) \Gamma_{0}$, it follows that

$$
\operatorname{ad}(\gamma) \Gamma_{0}=\bigcup\left\{\eta \operatorname{ad}(\beta) \Gamma_{0} \mid \eta \in \operatorname{ad}(\alpha) \Gamma_{0} / \operatorname{ad}(\alpha) \Gamma_{0} \cap \operatorname{ad}(\beta) \Gamma_{0}\right\}
$$

Thus, in $\Gamma / \Gamma_{0}, \quad R(\gamma)=\left|\operatorname{ad}(\alpha) \Gamma_{0} / \operatorname{ad}(\alpha) \Gamma_{0} \cap \operatorname{ad}(\beta) \Gamma_{0}\right| R(\beta)$, so $R(\gamma)\left|\operatorname{ad}(\alpha) \Gamma_{0} \cap \operatorname{ad}(\beta) \Gamma_{0}\right|=R(\alpha) R(\beta)$.

Proposition 3.2. Let $\left(\Gamma, \Gamma_{0}\right)$ be an almost normal subgroup pair. Suppose that $\alpha, \beta \in \mathcal{T}$, with $\alpha \vee_{s} \beta=\gamma$. Then $P_{[\alpha]} P_{[\beta]}=P_{[\gamma]}$, where $P_{[\alpha]}=W_{\alpha} W_{\alpha}^{*}$ and $\left[\right.$ ] denotes the equivalence class in $\mathcal{T} / N_{\Gamma_{0}}$.

Proof. Recall that $P_{[\alpha]}=W_{\alpha} W_{\alpha}^{*}=R(\alpha)^{-1} \sum\left\{\chi_{O_{\eta}} \mid \eta \in \operatorname{ad}(\alpha) \Gamma_{0}\right\}$ by Proposition 1.5, for $\alpha \in \mathcal{T}$. Thus $P_{[\alpha]} P_{[\beta]}=R(\alpha)^{-1} R(\beta)^{-1} \sum\left\{\chi_{O_{\eta}} \chi_{O_{\delta}}\right.$ $\left.\eta \in \operatorname{ad}(\alpha) \Gamma_{0}, \delta \in \operatorname{ad}(\beta) \Gamma_{0}\right\}=R(\alpha)^{-1} R(\beta)^{-1} \sum\left\{\chi_{O_{\eta} O_{\delta}} \mid \eta \in \operatorname{ad}(\alpha) \Gamma_{0}\right.$, $\left.\delta \in \operatorname{ad}(\beta) \Gamma_{0}\right\}$ the convolution product of functions in $H\left(\Gamma, \Gamma_{0}\right)$. The later 
expression

$$
\begin{gathered}
=R(\alpha)^{-1} R(\beta)^{-1} \sum\left\{\sum \left\{\sum\left\{\chi_{O_{\eta} \cdot O_{\delta}} \mid \delta \in \operatorname{ad}(\beta) \Gamma_{0}\right\} \mid\right.\right. \\
\left.\eta \in \operatorname{ad}(\alpha) \Gamma_{0} /\left(\operatorname{ad}(\beta) \Gamma_{0} \cap \operatorname{ad}(\alpha) \Gamma_{0}\right)\right\} \mid \\
\left.\eta \in \operatorname{ad}(\beta) \Gamma_{0} \cap \operatorname{ad}(\alpha) \Gamma_{0}\right\} \\
=R(\alpha)^{-1} R(\beta)^{-1} \sum\left\{R(\gamma) P_{[\gamma]} \mid \eta \in \operatorname{ad}(\beta) \Gamma_{0} \cap \operatorname{ad}(\alpha) \Gamma_{0}\right\} \\
=R(\alpha)^{-1} R(\beta)^{-1}\left|\operatorname{ad}(\beta) \Gamma_{0} \cap \operatorname{ad}(\alpha) \Gamma_{0}\right| R(\gamma) P_{[\gamma]}=P_{[\gamma]} \text { by Lemma 3.1. }
\end{gathered}
$$

Remark 3.3. If $\left(\Gamma, \Gamma_{0}\right)$ is an almost normal subgroup pair so that the solvable l.u.b. exists for any pair of elements in $\mathcal{T}$, and if $\pi: H\left(\Gamma, \Gamma_{0}\right) \rightarrow B(\mathcal{H})$ is a $*$-representation of $H\left(\Gamma, \Gamma_{0}\right)$, then Proposition 3.2 states that the map $\pi_{W}=\pi \circ W$ is a representation of the semigroup $\mathcal{T}$ satisfying the covariance condition

$$
\pi_{W}(\alpha) \pi_{W}(\alpha)^{*} \pi_{W}(\beta) \pi_{W}(\beta)^{*}=\pi_{W}\left(\alpha \vee_{s} \beta\right) \pi_{W}\left(\alpha \vee_{s} \beta\right)^{*}, \quad \alpha, \beta \in \mathcal{T}
$$

of Nica in $[\mathbf{N}]$.

Remark 3.4. For $\alpha, \beta \in \mathcal{T}$ we have that $\alpha^{-1} \vee_{s} \beta^{-1}=e$ if and only if either one of the two equivalent conditions $\alpha \beta \alpha^{-1} \vee_{s} \alpha=\alpha \beta, \beta \vee_{s} \beta \alpha \beta^{-1}=$ $\beta \alpha$ holds. In particular, if $\alpha \beta=\beta \alpha$ then $\alpha^{-1} \vee_{s} \beta^{-1}=e$ if and only if $\alpha \vee_{s} \beta=\alpha \beta$. Since $W_{\alpha} W_{\alpha}^{*} W_{\beta} W_{\beta}^{*}=W_{\alpha \vee_{s} \beta} W_{\alpha \vee_{s} \beta}^{*}$ by Proposition 3.2, we have $W_{\alpha}^{*} W_{\beta}=W_{\alpha}^{*} W_{\alpha} W_{a^{-1}\left(\alpha \vee_{s} \beta\right)}\left(W_{\beta} W_{\beta^{-1}\left(\alpha \vee_{s} \beta\right)}\right)^{*} W_{\beta}$, and so $W_{\alpha}^{*} W_{\beta}=$ $W_{\alpha^{-1}\left(\alpha \vee_{s} \beta\right)} W_{\beta^{-1}\left(\alpha \vee_{s} \beta\right)}$ which is equal to $W_{\beta} W_{\alpha}^{*}$ if $\alpha \beta=\beta \alpha$. Thus, for $\alpha, \beta \in \mathcal{T}$ with $\alpha^{-1} \vee_{x} \beta^{-1}=e$ and $\alpha \beta=\beta \alpha$ we have $W_{\alpha}^{*} W_{\beta}=W_{\beta} W_{\alpha}^{*}$.

Definition. For $\left(\Gamma, \Gamma_{0}\right)$ an almost normal subgroup pair, let $\mathcal{L}$ be the linear span over $\mathbb{C}$ of the set $\left\{W_{\alpha} W_{\beta}^{*} \mid \alpha, \beta \in \mathcal{T}\right\}$ in $H\left(\Gamma, \Gamma_{0}\right)$.

Proposition 3.5. Let $\left(\Gamma, \Gamma_{0}\right)$ be an almost normal subgroup pair and assume that the solvable l.u.b. exists for any pair of elements in $\mathcal{T}$. Then $\mathcal{L}$ is a $*$-subalgebra of $H\left(\Gamma, \Gamma_{0}\right)$.

Proof. Let $\alpha, \beta, \gamma, \delta \in \mathcal{T}$. Proposition 3.2 shows that $W_{\beta} W_{\beta}^{*} W_{\delta} W_{\delta}^{*}=$ $W_{\beta \vee_{s} \delta} W_{\beta \vee_{s} \delta}^{*}$. Thus

$$
\begin{aligned}
W_{\beta}^{*} W_{\delta} & =W_{\beta}^{*} W_{\beta \vee_{s} \delta} W_{\beta \vee_{s} \delta}^{*} W_{\delta} \\
& =W_{\beta}^{*} W_{\beta} W_{\beta^{-1}\left(\beta \vee_{s} \delta\right)}\left(W_{\delta} W_{\delta^{-1}\left(\beta \vee_{s} \delta\right)}\right)^{*} W_{\delta}=W_{\beta^{-1}\left(\beta \vee_{s} \delta\right)} W_{\delta^{-1}\left(\beta \vee_{s} \delta\right)}^{*}
\end{aligned}
$$

It follows that $W_{\alpha} W_{\beta}^{*} W_{\delta} W_{\gamma}^{*}=W_{\alpha \beta^{-1}\left(\beta \vee_{s} \delta\right)} W_{\gamma \delta^{-1}\left(\beta \vee_{s} \delta\right)}^{*}$, which is in $\mathcal{L}$. Clearly $\mathcal{L}$ is closed under adjoints. 
Lemma 3.6. Let $\left(\Gamma, \Gamma_{0}\right)$ be an almost normal subgroup pair so that the solvable l.u.b. exists for any pair of elements in $\mathcal{T}$ and that $\Gamma=\mathcal{T} T^{-1}=$ $\mathcal{T}^{-1} \mathcal{T}$. Suppose that $\alpha, \beta, \eta, \gamma \in \mathcal{T}$ satisfy $\alpha^{-1} \vee_{s} \beta^{-1}=\eta^{-1} \vee_{s} \delta^{-1}=e$.

(a) If $\alpha \beta^{-1}=\eta \delta^{-1}$ then there is an $n \in N_{\Gamma_{0}}$ with $\eta=\alpha$ and $\delta=\beta n$.

(b) If $O_{\alpha \beta^{-1}} \subseteq O_{\eta \delta^{-1}}$ then $[\alpha]=[\eta]$ and $[\beta]=[\delta]$ in $\mathcal{T} / N_{\Gamma_{0}}$.

Proof. (a) Set $x=\alpha \beta^{-1}$. Then $e \vee_{s} x=\alpha\left(\alpha^{-1} \vee_{s} \beta^{-1}\right)=\eta\left(\eta^{-1} \vee_{s} \delta^{-1}\right)$ by Lemma 2.2 and the comments preceding Theorem 2.3. Thus $\alpha$ and $\eta$ are both solvable l.u.b. for $e$ and $x$ so there is an $n \in N_{\Gamma_{0}}$ with $\alpha n=\eta$. Thus $\alpha \beta^{-1}=\alpha n \delta^{-1}$ and $\delta=\beta n$.

(b) Since $\Gamma_{0} \alpha \beta^{-1} \Gamma_{0} \subseteq \Gamma_{0} \eta \delta^{-1} \Gamma_{0} \subseteq \eta \Gamma_{0} \delta^{-1}$, there is an $m \in \Gamma_{0}$ with $\alpha \beta^{-1}=\eta m \delta^{-1}$. By part (a) there is an $n \in N_{\Gamma_{0}}$ with $\alpha=\eta m n$ and $\beta=\delta n$. Thus $[\alpha]=[\eta]$ and $[\beta]=[\delta]$ in $\mathcal{T} / N_{\Gamma_{0}}$.

Proposition 3.7. Let $\left(\Gamma, \Gamma_{0}\right)$ be an almost normal subgroup pair with $\Gamma=$ $\mathcal{T} T^{-1}=\mathcal{T}^{-1} \mathcal{T}$ and such that the solvable l.u.b. exists for any pair of elements of $\mathcal{T}$. Then $\mathcal{L}=H\left(\Gamma, \Gamma_{0}\right)$. If $N$ is a normal subgroup of $\Gamma$ with $\Gamma_{0}<N<N_{\Gamma_{0}}$ then $\mathcal{L}=\operatorname{span}_{\mathbb{C}}\left\{W_{s} W_{n} W_{t}^{*} \mid s^{-1} \vee_{s} t^{-1}=e, \quad n \in N / \Gamma_{0}\right.$ and $s, t \in \mathcal{F}\}$ where $\mathcal{F}$ is an arbitrarily chosen set in $\mathcal{T}$ of distinct coset representatives of $\mathcal{T} / N$.

Proof. That $\mathcal{L}=H\left(\Gamma, \Gamma_{0}\right)$ follows from Proposition 2.8. Let $\alpha, \beta \in \mathcal{T}$ be arbitrary. Then by Theorem 2.3 there are elements $s, t \in \mathcal{T}$ with $s=$ $e \vee_{s} \alpha \beta^{-1}=\alpha\left(\alpha^{-1} \vee_{s} \beta^{-1}\right)$ and $t=\beta\left(\alpha^{-1} \vee_{s} \beta^{-1}\right)$. Then $s^{-1} \vee_{s} t^{-1}=$ $\left(\alpha^{-1} \vee_{s} \beta^{-1}\right)^{-1}\left(\alpha^{-1} \vee_{s} \beta^{-1}\right)=e$. Since $\alpha=s\left(\alpha^{-1} \vee_{s} \beta^{-1}\right)^{-1}$ and $\beta=$ $t\left(\alpha^{-1} \vee_{s} \beta^{-1}\right)^{-1}$, it follows that $W_{\alpha} W_{\beta}^{*}=W_{s} W_{q}\left(W_{t} W_{q}\right)^{*}=W_{s} W_{q} W_{q}^{*} W_{t}^{*}$ where $q=\left(\alpha^{-1} \vee_{s} \beta^{-1}\right)^{-1} \in \mathcal{T}$. Since $N$ satisfies $\Gamma_{0} \unlhd N \unlhd \Gamma$, Proposition 1.5 and Theorem 1.7 show that $W_{q} W_{q}^{*}=R(q)^{-1} \sum\left\{U_{n} \mid n \in\left(\operatorname{ad}(q) \Gamma_{0}\right) / \Gamma_{0}\right\}$, a sum of $R(q)$ terms over $N / \Gamma_{0}$, and that $W_{\alpha} W_{\beta}^{*}$ is in the linear span over $\mathbb{Q}$ of $W_{s} W_{n} W_{t}^{*}$ where $n \in \operatorname{ad}(q) \Gamma_{0} \subseteq N$ and $s^{-1} \vee_{s} t^{-1}=e$. Note also that $(s n)^{-1} \vee_{s} t^{-1}=e$.

Proposition 3.8. Let $\left(\Gamma, \Gamma_{0}\right)$ be an almost normal subgroup pair with $\Gamma=$ $\mathcal{T} T^{-1}=\mathcal{T}^{-1} \mathcal{T}$ and such that the solvable l.u.b. exists for any pair of elements of $\mathcal{T}$. If $N_{\Gamma_{0}}$ is normal in $\Gamma$ then $\mathcal{B}=\left\{W_{s} W_{n} W_{t}^{*} \mid s^{-1} \vee_{s} t^{-1}=\right.$ e, $\left.\quad n \in N_{\Gamma_{0}} / \Gamma_{0}, s, t \in \mathcal{F}_{0}\right\}$ is a basis for $\mathcal{L}$. Here $\mathcal{F}_{0}$ is a set in $\mathcal{T}$ of coset representatives of $\mathcal{T} / N_{\Gamma_{0}}$.

Proof. The preceding proposition shows that this set is a spanning set for $\mathcal{L}$. By Lemma 2.6 it is enough to show that the elements $\left\{\chi_{O_{s n t-1}} \mid s, t \in\right.$ $\left.\mathcal{F}_{0}, s^{-1} \vee_{s} t^{-1}=e, n \in N_{\Gamma_{0}} / \Gamma_{0}\right\}$ are linearly independent in $H\left(\Gamma, \Gamma_{0}\right)$. If $O_{s n t^{-1}} \subseteq O_{p m q^{-1}}$ where $s, t, p, q \in F, s^{-1} \vee_{s} t^{-1}=p^{-1} \vee_{s} q^{-1}=e$ and $n, m \in$ $N_{\Gamma_{0}}$, then Lemma 3.6 (b) implies that $[s]=[s n]=[p m]=[p]$ in $\mathcal{T} / N_{\Gamma_{0}}$ and similarly $[t]=[q]$. Thus $s=p$ and $t=q$. Again, using Lemma 2.6 and the fact that $W_{s}$ and $W_{t}$ are isometries, we have that $W_{n}=W_{m}$ (up to a 
scalar), so $n=m$ in $N_{\Gamma_{0}} / \Gamma_{0}$. Since $\left\{O_{\gamma} \mid \gamma \in \Gamma\right\}$ are points in $\Gamma_{0} \backslash \Gamma / \Gamma_{0}$, this shows that the elements $\left\{\chi_{O_{s n t}-1} \mid s, t \in \mathcal{F}_{0}, s^{-1} \vee_{s} t^{-1}=e, n \in N_{\Gamma_{0}} / \Gamma_{0}\right\}$ are linearly independent.

In the presence of some more structure for the pair $\left(\Gamma, \Gamma_{0}\right)$ there is a slight strengthening of Proposition 3.8.

Proposition 3.9. Let $\left(\Gamma, \Gamma_{0}\right)$ be an almost normal subgroup pair with $\Gamma=$ $\mathcal{T} T^{-1}=\mathcal{T}^{-1} \mathcal{T}$ and such that the solvable l.u.b. exists for any pair of elements of $\mathcal{T}$. If $\Gamma_{0} \leq N \leq N_{\Gamma_{0}}$ with both $N$ and $N_{\Gamma_{0}}$ normal in $\Gamma$, then $\mathcal{B}_{N}=\left\{W_{s} W_{n} W_{t}^{*} \mid s^{-1} \vee_{s} t^{-1}=e, n \in N / \Gamma_{0}, s \in \mathcal{F}, t \in \mathcal{F}_{0}\right\}$ is a basis for $\mathcal{L}$. Here $\mathcal{F}_{0}$ is an arbitrary set in $\mathcal{T}$ of coset representatives of $\mathcal{T} / N_{\Gamma_{0}}$ and $\mathcal{F}$ is an arbitrary set in $\mathcal{T}$ of coset representatives of $\mathcal{T} / N$.

Proof. We show that there is a bijective correspondence between the set $\mathcal{B}_{N}$ and the basis $\mathcal{B}=\mathcal{B}_{N_{\Gamma_{0}}}$ of Proposition 3.8. First notice that given $s \in \mathcal{F}_{0}$ and $n \in N_{\Gamma_{0}}$ there is a unique $u \in \mathcal{F}$ and a unique $m \in N$ with $s n=u m$. To see this, observe $s n N=u N$ for a unique $u \in \mathcal{F}$. Then $s n \in u N$, so there is a unique $m \in N$ with $s n=u m$. We then have that the element $W_{s} W_{n} W_{t}^{*}$ of $\mathcal{B}$ is equal to the element $W_{s n} W_{t}^{*}=W_{u} W_{m} W_{t}^{*}$ of $\mathcal{B}_{N}$. Conversely, for $u \in \mathcal{F}$ and $m \in N$ given, there is a unique $s \in \mathcal{F}_{0}$ and $n \in N_{\Gamma_{0}}$ with $u m=s n$. This follows as before, by first noting that $u m N_{\Gamma_{0}}=s N_{\Gamma_{0}}$ for a unique $s \in \mathcal{F}_{0}$.

Theorem 3.10. Let $\left(\Gamma, \Gamma_{0}\right)$ be an almost normal subgroup pair with $\Gamma=$ $\mathcal{T}^{-1} \mathcal{T}=\mathcal{T} T^{-1}$, and such that the solvable l.u.b. exists for any pair of elements in $\mathcal{T}$. If $\Gamma_{0} \leq N \leq N_{\Gamma_{0}}$ with both $N$ and $N_{\Gamma_{0}}$ normal in $\Gamma$, then $H\left(\Gamma, \Gamma_{0}\right)$, respectively $C^{*}\left(\Gamma, \Gamma_{0}\right)$, is the universal $*$-algebra, respectively $C^{*}$ algebra, generated by $\left\{V_{\alpha} \mid \alpha \in \mathcal{T}\right\}$ such that

1) $V_{\alpha}^{*} V_{\alpha}=I=V_{n} \quad\left(\alpha \in \mathcal{T}, n \in \Gamma_{0}\right)$

2) $V_{\alpha} V_{\beta}=V_{\alpha \beta} \quad(\alpha, \beta \in \mathcal{T})$

3) $V_{\alpha} V_{\alpha}^{*} V_{\beta} V_{\beta}^{*}=V_{\alpha \vee_{s} \beta} V_{\alpha \vee_{s} \beta}^{*} \quad(\alpha, \beta \in \mathcal{T})$

4) $V_{\alpha} V_{n} V_{\alpha}^{*}=R(\alpha)^{-1} \sum\left\{V_{b} \mid b \in N / \Gamma_{0}\right.$ such that $\left[\operatorname{ad}\left(\alpha^{-1}\right) b\right]=[n]$ in $\left.N / \Gamma_{0}\right\},(\alpha \in \mathcal{T}, n \in N)$.

Proof. If $A$ is the universal *-algebra generated by $\left\{V_{\alpha} \mid \alpha \in \mathcal{T}\right\}$ with these relations, there is a natural $*$-homomorphism of $A$ to $H\left(\Gamma, \Gamma_{0}\right)$ mapping $V_{\alpha}$ to $W_{\alpha}$. This map is surjective since $\mathcal{L}=H\left(\Gamma, \Gamma_{0}\right)$ by Proposition 3.7. Define $\mathcal{L}^{\prime}$ to be the linear subspace of $A$ generated by $\left\{V_{\alpha} V_{\beta}^{*} \mid \alpha, \beta \in \mathcal{T}\right\}$. Using 1), 2) and 3), we see, as in Proposition 3.5, that $\mathcal{L}^{\prime}$ is a $*$-subalgebra of $A$. Since $\mathcal{L}^{\prime}$ contains the generators of $A, \mathcal{L}^{\prime}=A$. By 4), it follows as in Proposition 3.7, that $\left\{V_{s} V_{n} V_{t}^{*} \mid s^{-1} \vee_{s} t^{-1}=e, n \in N / \Gamma_{0}, \quad s, t \in \mathcal{F}\right\}$ spans $\mathcal{L}^{\prime}$. Now notice that condition 4) with $n=e$ implies that $V_{\alpha}$ is a unitary element of $A$ for $\alpha \in N_{\Gamma_{0}}$. For if $\alpha \in N_{\Gamma_{0}}$, then ad $(\alpha) \Gamma_{0}=\Gamma_{0}$ and ad $(\alpha)$ is an automorphism of $N_{\Gamma_{0}} / \Gamma_{0}$. The sum then has only $R(\alpha)=1$ terms, and $V_{b}$ for $b=e$ is the 
only term appearing. Thus $V_{\alpha} V_{\alpha}^{*}=I$ for $\alpha \in N_{\Gamma_{0}}$. Using this, we can show that the set $\left\{V_{u} V_{m} V_{w}^{*} \mid u^{-1} \vee_{s} w^{-1}=e, m \in N / \Gamma_{0}, u \in \mathcal{F}, w \in \mathcal{F}_{0}\right\}$ spans $\mathcal{L}^{\prime}$. To see this note that $t N_{\Gamma_{0}}=w N_{\Gamma_{0}}$ for some $w \in \mathcal{F}_{0}$, so $t=w p$ for some $p \in N_{\Gamma_{0}}$. Then $V_{s} V_{n} V_{t}^{*}=V_{s} V_{n}\left(V_{w} V_{p}\right)^{*}=V_{s} V_{n} V_{p^{-1}} V_{w}^{*}=V_{s n p^{-1}} V_{w}^{*}$. Now $s n p^{-1} N=u N$ for some $u \in \mathcal{F}$, so $s n p^{-1}=u m$ for some $m \in N$. Thus $V_{s} V_{n} V_{t}^{*}=V_{u} V_{m} V_{w}^{*}$ with $u \in \mathcal{F}, m \in N$ and $w \in \mathcal{F}_{0}$. Note also that $u^{-1} \vee_{s} w^{-1}=e$. Under the natural $*$-homomorphism above, the image of this spanning set in $H\left(\Gamma, \Gamma_{0}\right)$ is, by Proposition 3.9, linearly independent, so it must also be linearly independent in $A$, and so a basis for $A$. Thus $A \cong H\left(\Gamma, \Gamma_{0}\right)$.

We conclude this section by considering the case where $\Gamma$ is a semidirect product of the normal subgroup $N$ by $G, \Gamma_{0} \unlhd N$.

Theorem 3.11. Let $\left(\Gamma, \Gamma_{0}\right)$ be an almost normal subgroup pair with $e \rightarrow$ $N \rightarrow \Gamma \underset{\rho}{\stackrel{\nu}{\leftrightarrows}} G \rightarrow e$ a split exact sequence and $\Gamma_{0} \leq N \leq N_{\Gamma_{0}} \unlhd \Gamma$. Also assume that $G=T^{-1} T=T T^{-1}$ and that the solvable l.u.b. exists for any pair of elements of $T=\left\{g \in G \mid \Gamma_{0} \subseteq\right.$ ad $\left.(\nu(g)) \Gamma_{0}\right\}$. Then $H\left(\Gamma, \Gamma_{0}\right)$, respectively $C^{*}\left(\Gamma, \Gamma_{0}\right)$ is the universal $*$-algebra, respectively $C^{*}$-algebra, generated by $\left\{V_{g}, U_{n} \mid g \in T, n \in N / \Gamma_{0}\right\}$ such that for $g, h \in T, n, m \in N / \Gamma_{0}$

1) $V_{g}^{*} V_{g}=I$

2) $V_{g} V_{h}=V_{g h}$

3) $V_{g} V_{g}^{*} V_{h} V_{h}^{*}=V_{g \vee_{s} h} V_{g \vee_{s} h}^{*}$

4) $U_{n m}=U_{n} U_{m}$ and $U_{n}^{*}=U_{n^{-1}}, U_{e}=V_{e}$

5) $U_{n} V_{g}=V_{g} U_{\mathrm{ad}}\left(v\left(g^{-1}\right)\right) n$

6) $V_{g} U_{n} V_{g}^{*}=R(g)^{-1} \sum\left\{U_{b} \mid \operatorname{ad}\left(\nu\left(g^{-1}\right)\right) b=n\right\}$.

Proof. First note that $V_{e}^{2}=V_{e}$ by 2), so $I=V_{e}^{*} V_{e}=V_{e}^{*} V_{e} V_{e}=V_{e}$. Note that the first three conditions state that $V$ is a covariant representation by "isometries" of the semigroup $T$, condition 4) states that $U$ is a "unitary" representation of the group $N / \Gamma_{0}$ while condition 6$)$ is saying that $(U, V)$ is a "covariant pair". We prove this result directly, rather than using Theorem 3.10. Let $A$ be the universal *-algebra generated by the $V_{g}$ and $U_{n}$ subject to the six conditions. Define $\mathcal{L}^{\prime}=\operatorname{span}_{\mathbb{C}}\left\{V_{s} U_{n} V_{t}^{*} \mid s, t \in T, n \in N / \Gamma_{0}\right\}$. We claim that $\mathcal{L}^{\prime}$ is a $*$-subalgebra of $A$, thus, since it contains the generators of $A, A=\mathcal{L}^{\prime}$. To see this it is enough to show that $V_{s} U_{n} V_{t}^{*} V_{u} U_{m} V_{w}^{*}$ is of the form $V_{a} U_{p} V_{b}^{*}$. Condition 3) states $V_{t} V_{t}^{*} V_{u} V_{u}^{*}=V_{t \vee_{s} u} V_{t \vee_{s} u}^{*}$, so by 1), $V_{t}^{*} V_{u}=$ $V_{t}^{*} V_{t \vee_{s} u} V_{t \vee_{s} u}^{*} V_{u}=V_{t}^{*} V_{t} V_{t^{-1}\left(t \vee_{s} u\right)} V_{u^{-1}\left(t \vee_{s} u\right)}^{*} V_{u}^{*} V_{u}=V_{t^{-1}\left(t \vee_{s} u\right)} V_{u^{-1}\left(t \vee_{s} u\right)}^{*}$. Substituting this into $V_{s} U_{n} V_{t}^{*} V_{u} U_{m} V_{w}^{*}$ and using condition 5) and its adjoint, finishes the claim.

One can define a $*$-homomorphism of $A$ to $H\left(\Gamma, \Gamma_{0}\right)$ by mapping $V_{g}$ to $W_{\nu(g)}$ and $U_{n}$ to $W_{n}$, since the six conditions are straightforward to verify for $W_{\nu(g)}$ and $W_{n}$. 
The argument of Proposition 3.7 using condition 6) shows that $\mathcal{L}^{\prime}=$ $\operatorname{span}_{\mathbb{C}}\left\{V_{s} U_{n} V_{t}^{*} \mid s^{-1} \vee_{s} t^{-1}=e, s, t \in T, n \in N / \Gamma_{0}\right\}$. The argument in Theorem 3.10 using condition 6 ) with $n=e$ shows that $\mathcal{L}^{\prime}=\operatorname{span}_{\mathbb{C}}\left\{V_{s} U_{n} V_{t}^{*} \mid\right.$ $\left.s^{-1} \vee_{s} t^{-1}=e, s \in T, n \in N / \Gamma_{0}, t \in \mathrm{F}_{0}\right\}$ where $\mathrm{F}_{0}$ is a set in $T$ of coset representatives of $T / \rho\left(N_{\Gamma_{0}}\right)$. The image of this spanning set in $H\left(\Gamma, \Gamma_{0}\right)$ is a basis in $H\left(\Gamma, \Gamma_{0}\right)$ by Proposition 3.9 , so $A \cong H\left(\Gamma, \Gamma_{0}\right)$.

Within the context of the preceding theorem, we saw earlier in Theorem 2.11 that there is a natural surjective $*$-homomorphism of $C^{*}\left(N / \Gamma_{0}\right) \rtimes T$ onto $C^{*}\left(\Gamma, \Gamma_{0}\right)$. In fact this map is an isomorphism.

Theorem 3.12. Let $\left(\Gamma, \Gamma_{0}\right)$ be an almost normal subgroup pair with $e \rightarrow$ $N \rightarrow \Gamma \stackrel{\nu}{\leftrightarrows} G \rightarrow$ e a split exact sequence. Assume also that $\Gamma_{0} \leq N \leq N_{\Gamma_{0}} \unlhd$ $\Gamma, G=T^{-1} T=T T^{-1}$ and that the solvable l.u.b. exists for any pair of elements of $T=\left\{g \in G \mid \Gamma_{0} \subseteq \operatorname{ad}(\nu(g)) \Gamma_{0}\right\}$. Then the natural surjective *-homomorphism of $C^{*}\left(N / \Gamma_{0}\right) \rtimes T$ onto $C^{*}\left(\Gamma, \Gamma_{0}\right)$ is an isomorphism.

Proof. As in Section 1, for $n \in N / \Gamma_{0}$ let $\delta_{n}$ be the element of $l^{1}\left(N / \Gamma_{0}\right)$ which is one at $n$ and zero elsewhere. Let $\eta: l^{1}\left(N / \Gamma_{0}\right) \rightarrow C^{*}\left(N / \Gamma_{0}\right)$ be the natural norm decreasing injective $*$-homomorphism and $u_{n}=\eta\left(\delta_{n}\right)$ the natural unitaries generating $C^{*}\left(N / \Gamma_{0}\right)$. Also denote by $\left\{Y_{g} \mid g \in T\right\}$ the semigroup of isometries in $C^{*}\left(N / \Gamma_{0}\right) \rtimes T$ implementing the action of the semigroup $T$ in the dynamical system $\left(C^{*}\left(N / \Gamma_{0}\right), \tilde{\Theta}, T\right)$ described before Theorem 1.10.

Define $V_{g}=Y_{g}$ and $U_{n}=u_{n}$ for $g \in T$ and $n \in N / \Gamma_{0}$. We first show that the conditions of Theorem 3.11 are fulfilled. It is clear that conditions $1), 2), 4)$, and 6) hold for this family of elements in $C^{*}\left(N / \Gamma_{0}\right) \rtimes T$. Indeed, these are the defining relations for $C^{*}\left(N / \Gamma_{0}\right) \rtimes T$. We only need to show that conditions 3) and 5) also hold. By condition 6), $V_{g} V_{g}^{*}=Y_{g} Y_{g}^{*}=$ $R(g)^{-1} \sum\left\{U_{b} \mid \operatorname{ad}\left(\nu\left(g^{-1}\right)\right) b=e\right\}=\eta\left[R(g)^{-1} \sum\left\{\delta_{b} \mid b \in \operatorname{ad}(\nu(g)) \Gamma_{0}\right.\right.$, distinct in $\left.\left.N / \Gamma_{0}\right\}\right]$. The argument of Proposition 3.2 along with the fact that $\eta$ is a $*$-homomorphism shows that condition 3 ) holds.

We now check condition 5). First note that $V_{g} V_{g}^{*}=\eta\left(\tilde{\Theta}_{g}\left(\delta_{e}\right)\right)$ by condition 6). Thus $U_{n} V_{g}=\eta\left(\delta_{n}\right) V_{g}=\eta\left(\delta_{n}\right) Y_{g} Y_{g}^{*} Y_{g}=\eta\left(\delta_{n} * \tilde{\Theta}_{g}\left(\delta_{e}\right)\right) Y_{g}$. Also $V_{g} U_{\text {ad } g^{-1}(n)}=Y_{g} \eta\left(\delta_{\text {ad } g^{-1}(n)}\right)=Y_{g} \eta\left(\delta_{\text {ad } g^{-1}(n)}\right) Y_{g}^{*} Y_{g}=\eta\left(\tilde{\Theta}_{g}\left(\delta_{\text {ad } g^{-1}(n)}\right)\right) Y_{g}$ by condition 6$)$. It is a straightforward calculation to check that the convolution product $\delta_{n} * \tilde{\Theta}_{g}\left(\delta_{e}\right)=\tilde{\Theta}_{g}\left(\delta_{\operatorname{ad} g^{-1}(n)}\right)$, so condition 5) is verified.

Theorem 3.11 yields a $*$-homomorphism of $C^{*}\left(\Gamma, \Gamma_{0}\right)$ to $C^{*}\left(N / \Gamma_{0}\right) \rtimes T$ which is easily seen to be surjective and the inverse of the surjective $*$ homomorphism of Theorem 2.11. 


\section{Examples.}

In this section we illustrate and apply some of the above results to various examples. As we will see, many of the examples given are special cases of other examples.

4.1.

As a first example, it is illustrative to see the Bost-Connes context. Their work provides the framework for much of this work. Here $\Gamma$ is the semidirect product of the abelian group $N=(\mathbb{Q},+)$ and the abelian group $G=\left(\mathbb{Q}_{+}^{\times}, \cdot\right)$, the multiplicative group of nonzero positive rational numbers. The action $\psi: G \rightarrow \operatorname{Aut}(N)$ is given by the ring structure of $\mathbb{Q}$, namely $\psi_{g}(r)=g r$ for $g \in G, r \in N$. For a subgroup $\Gamma_{0}$ of $N$ to be an almost normal subgroup of $\Gamma$, it is only necessary by Lemma 1.9 that the subgroup $\psi_{g}\left(\Gamma_{0}\right) \Gamma_{0} / \Gamma_{0}$ of $N / \Gamma_{0}$ is finite for each $g \in G$. If $\Gamma_{0}=\mathbb{Z}$, for $g=a b^{-1}$ with $a, b \in \mathbb{N}, b \neq 0$, we have $\left(\psi_{g} \mathbb{Z}+\mathbb{Z}\right) / \mathbb{Z} \cong\left(\psi_{a} \mathbb{Z}+\psi_{b} \mathbb{Z}\right) / \psi_{b} \mathbb{Z}=(a, b) \mathbb{Z} / b \mathbb{Z}$, a finite group. Since $\psi_{g}\left(\Gamma_{0}\right) \neq \Gamma_{0}$ for all $g \neq e$ in $G, N$ is the normalizer $N_{\Gamma_{0}}$ of $\Gamma_{0}$ in $\Gamma$. We compute that $T^{-1}=\left\{g \in G \mid \psi_{g}(\mathbb{Z}) \subseteq \mathbb{Z}\right\}=\mathbb{N}$ and so $G=T^{-1} T=T T^{-1}$. For $a=n^{-1}, b=m^{-1}$ in $T=\mathbb{N}^{-1}$, we have $a \leq b$ if and only if $n \mid m$, so $a \vee_{s} b$ exists and is the least common multiple of $n$ and $m$. Thus $a^{-1} \vee_{s} b^{-1}=e$ if and only if $n, m$ are relatively prime. The $C^{*}$-Hecke algebra $C^{*}\left(\Gamma, \Gamma_{0}\right)$ is thus isomorphic to the semigroup crossed product $C^{*}$-algebra $C^{*}(\mathbb{Q} / \mathbb{Z}) \rtimes T$ $\cong C^{*}(\mathbb{Q} / \mathbb{Z}) \rtimes \mathbb{N}$, where we use the isomorphism of $G$ given by $n \rightarrow n^{-1}$, mapping the semigroup $T$ to $\mathbb{N}$.

\section{2.}

With $N, G$ as in Example 4.1, there are different possible actions $\psi$ : $G \rightarrow \operatorname{Aut}(N)$, each giving rise to a split extension $\Gamma_{\psi}$ of $N$ by $G$. The group $\operatorname{Aut}(N)=G L(1, \mathbb{Q})=\left(\mathbb{Q}^{\times}, \cdot\right)$, so $\psi$ is determined by its effect on $P$, the prime members of $\mathbb{N}$, since $\left(\mathbb{Q}_{+}^{\times}, \cdot\right) \cong \bigoplus_{P} \mathbb{Z}$. If both $\psi^{-1}(1)$ and $\psi^{-1}(-1)$ are contained in $\{1\}$, the normalizer $N_{\Gamma_{0}}$ of $\Gamma_{0}=\mathbb{Z}$ is $N$ and a similar analysis to that of 4.1 may be carried through.

\section{3.}

For $d \in \mathbb{N}$, let $G=\{g \in G L(d, \mathbb{Q}) \mid \operatorname{det} g>0\}=G L(d, \mathbb{Q})_{+}$and $N=\left(\mathbb{Q}^{d},+\right)$, with $\psi: G \rightarrow \operatorname{Aut}(N)$ the inclusion map. Setting $\Gamma$ to be an extension of $N$ by $G$ and $\Gamma_{0}=\mathbb{Z}^{d}$ we check that $\left(\Gamma, \Gamma_{0}\right)$ is an almost normal subgroup pair. Again we need only check that $\psi_{g}\left(\mathbb{Z}^{d}\right)+\mathbb{Z}^{d} / \mathbb{Z}^{d}$ is finite for $g \in G$. Choosing $g \in G$, there is an $m \in \mathbb{N}$ with $m g \in \mathfrak{M}_{d}(\mathbb{Z})$. For example, there is an $m \in \mathbb{N}$ so that the ideal $\left\{r \in \mathbb{Z} \mid r g \in \mathfrak{M}_{d}(\mathbb{Z})\right\}$ of $\mathbb{Z}$ is $m \mathbb{Z}$. Then $\psi_{g}\left(\mathbb{Z}^{d}\right)+\mathbb{Z}^{d} / \mathbb{Z}^{d}=\psi_{m^{-1}} \psi_{m g}\left(\mathbb{Z}^{d}\right)+Z^{d} / \mathbb{Z}^{d} \cong \psi_{m g} \mathbb{Z}^{d}+$ $\psi_{m} \mathbb{Z}^{d} / \psi_{m} \mathbb{Z}^{d} \subseteq \mathbb{Z}^{d} / \psi_{m}\left(\mathbb{Z}^{d}\right)$, which has $m^{d}$ elements. The semigroup $T^{-1}=$ $\left\{g \in G \mid \psi_{g}\left(\mathbb{Z}^{d}\right) \subseteq \mathbb{Z}^{d}\right\}=\mathfrak{M}_{d}(\mathbb{Z}) \cap G$, and $T \cap T^{-1}=S L(d, \mathbb{Z})$, which is not normal in $G$. Also $T T^{-1}=T^{-1} T=G$ and $a \vee_{s} b$ exists for each $a, b \in$ $T$. Applying Proposition 2.8 gives us the universal $C^{*}$-algebra $C^{*}\left(\Gamma, \Gamma_{0}\right)$. Theorem 2.11 also applies. 


\section{4 .}

This example is a special case of Example 4.3, but designed to circumvent the problem of $N_{\Gamma_{0}}$ not being normal in $\Gamma$. It is also the example which motivated my work in this paper, cf. [B].

Choose $M, F \in \mathfrak{M}_{d}(\mathbb{Z})$ with $M F=F M$, $\operatorname{det} F$ and $\operatorname{det} M$ both nonzero and relatively prime. Define an action $\psi: \mathbb{Z} \oplus \mathbb{Z} \rightarrow$ Aut $(N)$ by $\psi(n, m)=$ $F_{t}^{-n} M_{t}^{-m}$ where $N$ is the subgroup of $\mathbb{Q}^{d}$, endowed with the discrete topology, generated by $\left\{F_{t}^{n} M_{t}^{m}\left(\mathbb{Z}^{d}\right) \mid n, m \in \mathbb{Z}\right\}$. With $\Gamma$ chosen so that $e \rightarrow$ $N \rightarrow \Gamma \rightarrow \mathbb{Z} \oplus \mathbb{Z} \rightarrow e$ is an extension inducing the given $\psi$, and with $\Gamma_{0}=\mathbb{Z}^{d}$, the argument of Example 4.3) shows that $\left(\Gamma, \Gamma_{0}\right)$ is an almost normal subgroup pair. The semigroup $T=\left\{(n, m) \in \mathbb{Z} \oplus \mathbb{Z} \mid F_{t}^{n} M_{t}^{m}\left(\mathbb{Z}^{d}\right) \subseteq \mathbb{Z}^{d}\right\}$ contains $\mathbb{N} \oplus \mathbb{N}$ and $T \cap T^{-1}=\left\{(n, m) \in \mathbb{Z} \oplus \mathbb{Z} \mid F_{t}^{n} M_{t}^{m} \in G L(d, \mathbb{Z})\right\}$. Recall that $(\operatorname{det} F, \operatorname{det} M)=1$, so if we stipulate that both $|\operatorname{det} F|$ and $|\operatorname{det} M|$ are not 1 , then $(n, m) \in T \cap T^{-1}$ if and only if $n=m=0$. It also follows in this situation that $\mathbb{N} \oplus \mathbb{N}=T$. Thus $T T^{-1}=T^{-1} T=\mathbb{Z} \oplus \mathbb{Z}$. We now show that any pair of elements of the additive group $G=\mathbb{Z} \oplus \mathbb{Z}$ has a solvable l.u.b. The argument of Proposition 3.11 of $[\mathbf{B}]$ shows that $F^{a} \mathbb{Z}^{d}+M^{b} \mathbb{Z}^{d}=\mathbb{Z}^{d}$ for $a, b \in \mathbb{N}$. If we denote the minimum of two integers $a$ and $b$ by $a \wedge b$, it follows, since $M$ and $F$ commute, that $F^{a} \mathbb{Z}^{d}+M^{b} \mathbb{Z}^{d}=$ $F^{a \wedge 0} M^{b \wedge 0}\left(\mathbb{Z}^{d}\right)$. Thus, for $(n, m),(p, q) \in G, F^{n} M^{m}\left(\mathbb{Z}^{d}\right)+F^{p} M^{q}\left(\mathbb{Z}^{d}\right)=$ $F^{n \wedge p} M^{m \wedge q}\left[F^{n-n \wedge p} M^{m-m \wedge q}\left(\mathbb{Z}^{d}\right)+F^{p-n \wedge p} M^{q-m \wedge q}\left(\mathbb{Z}^{d}\right)\right]=F^{n \wedge p} M^{m \wedge q}\left(\mathbb{Z}^{d}\right)$, so $(n, m) \vee_{s}(p, q)=(n \wedge p, m \wedge q)$. Applying Theorem 3.10 we have, for $\Gamma$ the semidirect product of $N$ by $\mathbb{Z} \oplus \mathbb{Z}$ with respect to $\psi$, that the Hecke $C^{*}$-algebra $C^{*}\left(\Gamma, \Gamma_{0}\right)$ is isomorphic to the semigroup crossed product $C^{*}$ algebra $C^{*}\left(N / \Gamma_{0}\right) \rtimes T=C^{*}\left(N / \Gamma_{0}\right) \rtimes(\mathbb{N} \oplus \mathbb{N})$. There are other similar examples along these lines.

\section{5.}

Let $K$ denote an algebraic number field; so a subfield of $\mathbb{C}$ which is a finite dimensional extension of $\mathbb{Q}$. Thus $K=\mathbb{Q}[\alpha]$ for some algebraic number $\alpha$, say of degree $d$. Letting $\Gamma_{0}$ denote the ring of algebraic integers in $K$, choose an integral basis $B=\left\{\beta_{1}, \ldots, \beta_{d}\right\}$ of $\Gamma_{0}$; so $\Gamma_{0}$ is the $\mathbb{Z}$-module generated by $B$. Let $\eta$ denote the norm for $K$ over $\mathbb{Q}$. The group of units of the ring $\Gamma_{0}$, denoted by $\mathcal{U}$, is $\left\{g \in \Gamma_{0} \mid \eta(g)= \pm 1\right\}$ and $K^{\times}$denotes the multiplicative group of units of $K$. There is an action $\psi: K^{\times} \rightarrow \operatorname{Aut}(K)$, where $K$ is viewed as an additive abelian group, given by $\psi_{g}(k)=g k, k \in K$ and $g \in K^{\times}$. Also set $T^{-1}=\left\{g \in K^{\times} \mid \psi_{g}\left(\Gamma_{0}\right) \subseteq \Gamma_{0}\right\}$. Since $1 \in \Gamma_{0}$, the condition $\psi_{g} \Gamma_{0} \subseteq \Gamma_{0}$ holds if and only if $g \in \Gamma_{0}$, so $T^{-1}=\Gamma_{0} \cap K^{\times}=\Gamma_{0}^{\times}$.

Lemma 4.5.1. $K^{\times}=T^{-1} T=T T^{-1}$.

Proof. For $g \in K^{\times}$there is an $m \in \mathbb{N} /\{0\}$ with $m g \in \Gamma_{0}$, so $\psi_{m g}\left(\Gamma_{0}\right) \subseteq \Gamma_{0}$ and $m g \in T^{-1}$. Since $m \in T^{-1}$ it follows that $g=m^{-1}(m g)=(m g) m^{-1} \in$ $T T^{-1} \cap T^{-1} T$. 
The argument of Example 4.3 shows that $\left(\psi_{g}\left(\Gamma_{0}\right)+\Gamma_{0}\right) / \Gamma_{0}$ is finite for $g \in K^{\times}$, so $\left(\Gamma, \Gamma_{0}\right)$ is an almost normal subgroup pair whenever $e \rightarrow K \rightarrow$ $\Gamma \rightarrow K^{\times} \rightarrow e$ is an extension inducing the action $\psi$.

Lemma 4.5.2. $T \cap T^{-1}=\mathcal{U}$.

Proof. If $g \in T \cap T^{-1}$, then $\psi_{g} \Gamma_{0}=\Gamma_{0}$, so $\psi_{g}$ as a matrix with respect to the basis $B$ of $\Gamma_{0}$, is in $G L(d, \mathbb{Z})$. Thus $\operatorname{det}\left(\psi_{g}\right)= \pm 1$. Since $\eta(g)=\operatorname{det}\left(\psi_{g}\right)$, we have $g \in \mathcal{U}$. Conversely, if $g$ is a unit of $\Gamma_{0}$, then $\psi_{g}\left(\Gamma_{0}\right)=\Gamma_{0}$.

To be able to continue with this example, we need to show that solvable least upper bounds exist for pairs of elements from $T$. Since $K^{\times}$is an abelian group, this is equivalent to any pair of elements from $T^{-1}$ possessing a solvable l.u.b. For $g, h \in T^{-1}, g, h$ also belong to $\Gamma_{0}$, so the subgroup $\psi_{g} \Gamma_{0}+\psi_{h} \Gamma_{0}$ is the sum of two principle ideals in the ring $\Gamma_{0}$, so also an ideal. The question of whether the solvable l.u.b. of $g$ and $h$ exists in $T^{-1}$ is then equivalent to whether every ideal of $\Gamma_{0}$ is principle. For if $c \in \Gamma_{0}$ with $\psi_{g} \Gamma_{0}+\psi_{h} \Gamma_{0}=\psi_{c} \Gamma_{0}$ then $\operatorname{rank}\left(\psi_{c} \Gamma_{0}\right) \geq \operatorname{rank}\left(\psi_{g} \Gamma_{0}\right) \geq d$. Thus $\eta(c) \neq 0$ and $c \in \Gamma_{0} \cap K^{\times}=T^{-1}$. Since every ideal of $\Gamma_{0}$ can be written as the sum of two principal ideals, the equivalence is established. Assuming then that every ideal of $\Gamma_{0}$ is equivalent to a principle ideal, namely that the class group of the field $K$ consists of the unit element only, i.e., that the class number $h_{K}$ of $K$ is 1 , we have that solvable least upper bounds exist in $T^{-1}$.

Remark 4.5.3. The subgroup $K_{+}^{\times}=\{g \in K \mid \eta(g)>0\}$ of $K^{\times}$is just $\mathbb{Q}_{+}$when $K=\mathbb{Q}$, which is the group considered in $[\mathbf{B C}]$. One could try the same approach as above in this situation, obtaining for example $T^{-1}=$ $\Gamma_{0} \cap K_{+}^{\times}$and $K_{+}^{\times}=T T^{-1}=T^{-1} T$; however, there are examples where $\psi_{g} \Gamma_{0}+\psi_{h} \Gamma_{0}=\psi_{c} \Gamma_{0}$ with $g, h \in T^{-1}$, so $\eta(g)$ and $\eta(h)$ are both positive, but $\eta(c)<0$ for any such $c$, so $c \notin T^{-1}$. As an example, consider $K=Q[\sqrt{6}]$. Then $\Gamma_{0}=\mathbb{Z}[\sqrt{6}]$ and $\mathcal{U}$, the units of $\Gamma_{0}$, are $\left\{ \pm(5+2 \sqrt{6})^{n} \mid n \in \mathbb{Z}\right\}$. These units all have norm 1 . Setting $g=2$ and $h=4+\sqrt{6}$ we have that the norms of $g$ and $h$ are both positive, equal to 4 and 10 respectively. Now write $g=(2+\sqrt{6})^{2}(5-2 \sqrt{6})$ and $h=(2+\sqrt{6})(-1+\sqrt{6})$. Since $\eta(2+\sqrt{6})=-2$, the element $2+\sqrt{6}$ of $\Gamma_{0}$ is indecomposable. Also $\eta(-1+\sqrt{6})=-5$, so $-1+\sqrt{6}$ is indecomposable. Since $2+\sqrt{6}=-g+h$, it follows that $\psi_{g} \Gamma_{0}+\psi_{h} \Gamma_{0}=\psi_{c} \Gamma_{0}$ where $c=2+\sqrt{6}$ is an element of negative norm. If $c \Gamma_{0}=d \Gamma_{0}$ then $d$ must be $c$ up to multiplication by a unit of $\Gamma_{0}$, so $\eta(c)<0$ for any $c \in \Gamma_{0}$ with $\psi_{g} \Gamma_{0}+\psi_{h} \Gamma_{0}=\psi_{c} \Gamma_{0}$. We also mention that $\Gamma_{0}$ is Euclidean and so a principle ideal domain. Thus $h_{K}=1$ for this example.

It is a straightforward computation using that $K^{\times}$is abelian to show that $N_{\Gamma_{0}}$ is a normal subgroup of $\Gamma$, so Theorem 3.12 applies. 
Proposition 4.5.4. Let $K$ be a number field with class number 1. Denoting the ring of algebraic integers by $\Gamma_{0},\left(\Gamma, \Gamma_{0}\right)$ is an almost normal subgroup pair where $\Gamma$ is the semidirect product $K \rtimes_{\psi} K^{\times}$with $\psi: K^{\times} \rightarrow \operatorname{Aut}(K)$ given by multiplication. If $T=\left\{g \in K^{\times} \mid \Gamma_{0} \subseteq \psi_{g}\left(\Gamma_{0}\right)\right\}$ then $T^{-1}=\Gamma_{0} \cap K^{\times}$, the Hecke algebra $H\left(\Gamma, \Gamma_{0}\right)$ has a universal $C^{*}$-norm, and there is a natural *isomorphism of the $C^{*}$-semigroup crossed product algebra $C^{*}\left(K / \Gamma_{0}\right) \rtimes T$ with $C^{*}\left(\Gamma, \Gamma_{0}\right)$, the $C^{*}$-completion of $H\left(\Gamma, \Gamma_{0}\right)$. If $\Gamma_{0}$ has a unit of norm -1 , the statement remains true if $K^{\times}$is replaced with $K_{+}^{\times}=\left\{g \in K^{\times} \mid \eta(g)>0\right\}$ and $T=\left\{g \in K_{+}^{\times} \mid \Gamma_{0} \subseteq \psi_{g}\left(\Gamma_{0}\right)\right\}$.

Consider the multiplicative group $\mathcal{J}$ of fractional ideals of the Dedekind domain $\Gamma_{0}$. This is a free abelian group generated by the prime ideals of $\Gamma_{0}$, with unit element the ideal $\Gamma_{0}$. A fractional ideal is of the form $d^{-1} J$ for some integral ideal $J$ of $\Gamma_{0}$ and some $d \neq 0$ in $\Gamma_{0}$. The fractional principle ideals $k \Gamma_{0}$ with $k \in K^{\times}$form a subgroup $\mathcal{K}$ of $\mathcal{J}$, namely the image of the group homomorphism $\varphi: K^{\times} \rightarrow \mathcal{J}$ mapping $k$ to $k \Gamma_{0}$. The kernel of this homomorphism is $\mathcal{U}$, the group of units of $\Gamma_{0}$, so $K^{\times} / \mathcal{U}$ is isomorphic to the subgroup $\mathcal{K}$ of fractional principle ideals of $\mathcal{J}$. Note that if the class number of $K$ is 1 , all ideals of $\Gamma_{0}$ are principle, so $\varphi$ is surjective. Since $\mathcal{J}$ is a free abelian group, so is the subgroup of fractional principal ideals $K$ and therefore the exact sequence $e \rightarrow \mathcal{U} \rightarrow K^{\times} \rightarrow \mathcal{K} \rightarrow e$ splits, yielding a subgroup $\mathcal{H}$ of $K^{\times}$, isomorphic with $\mathcal{K}$ and with $\mathcal{H} \cap \mathcal{U}=\{e\}, \mathcal{H} \oplus \mathcal{U} \cong K^{\times}$.

Theorem 4.5.5. Let $K$ be a number field with class number $1, \Gamma_{0}$ the ring of algebraic integers in $K$. Then $\left(\Gamma, \Gamma_{0}\right)$ is an almost normal subgroup pair where $\Gamma$ is the semidirect product $K \rtimes_{\psi} \mathcal{H}$ with $\mathcal{H}$ a subgroup of $K^{\times}$complementing $\mathcal{U}$, the group of units of $\Gamma_{0}$ and $\psi: \mathcal{H} \rightarrow$ Aut $(K)$ defined by multiplication: $\psi_{g}(h)=g k, g \in \mathcal{H}, k \in K$. If $P=\left\{g \in \mathcal{H} \mid \Gamma_{0} \subseteq \psi_{g}\left(\Gamma_{0}\right)\right\}$ then the Hecke algebra $H\left(\Gamma, \Gamma_{0}\right)$ has a universal $C^{*}$-norm, and there is a natural *-isomorphism of the $C^{*}$-semigroup crossed product algebra $C^{*}\left(K / \Gamma_{0}\right) \rtimes P$ with $C^{*}\left(\Gamma, \Gamma_{0}\right)$, the $C^{*}$-completion of $H\left(\Gamma, \Gamma_{0}\right)$.

4.6.

It seems worthwhile to include another example, as it encompasses all of the examples mentioned above and uses standard constructions in ring theory $([\mathbf{R}])$. Let $R$ be a unital ring and $\Gamma_{0}$ an $R$-module. For example, if $\Gamma_{0}$ is an abelian group, it can be viewed as an $R$-module where $R$ is any unital subring of the ring $\mathcal{R}=\operatorname{Hom}_{\mathbb{Z}}\left(\Gamma_{0}, \Gamma_{0}\right)$ by setting $f \cdot m=f(m)$ for $f \in \mathcal{R}, m \in \Gamma_{0}$. In general, the left regular representation of $R$ is a ring homomorphism $\rho: R \rightarrow \mathcal{R}$.

Now choose a unital multiplicatively closed subset $S$ of the center $Z(R)$ of $R$ and form $N$, the localization of the module $\Gamma_{0}$ at $S, N=S^{-1} \Gamma_{0}$. One construction of $N$ involves considering $S$ as a preordered directed set under $s \leq t$ if and only if $s$ divides $t$. Define $\varphi_{s}^{t}: \Gamma_{s} \rightarrow \Gamma_{t}$ for $s \leq t$ by restriction, 
where $\Gamma_{s}$ is the abelian group $\operatorname{Hom}_{R}\left(R s, \Gamma_{0}\right)$ and set $S^{-1} \Gamma_{0}=\lim _{-}\left(\Gamma_{s}, \varphi_{s}^{t}\right)$, a limit of $\mathbb{Z}$-modules. If $S^{-1} R$ denotes the ring obtained by localizing the ring $R$ at $S$, then $N$ becomes an $S^{-1} R$ module. Letting $G$ denote the group of units of the ring $S^{-1} R$ we have $S \subseteq G$ and we obtain an action of the group $G$ which extends the original action of $S$ on $\Gamma_{0}$. This construction is basically the one Cuntz used in forming the crossed product of a $C^{*}$-algebra by an endomorphism $[\mathbf{C}]$.

Lemma 4.6.1. If $\Gamma_{0} / s \Gamma_{0}$ is finite for each $s \in S$ then $\left(\Gamma, \Gamma_{0}\right)$ is an almost normal subgroup pair for any extension $e \rightarrow N \rightarrow \Gamma \rightarrow G \rightarrow e$.

Proof. Using Lemma 1.9 it suffices to show that $g \Gamma_{0}+\Gamma_{0} / \Gamma_{0}$ is finite for each $g \in G$. Writing $g=s^{-1} r$ for some $s \in S, r \in R$ we have $g \Gamma_{0}+\Gamma_{0} / \Gamma_{0} \cong$ $\left(r \Gamma_{0}+s \Gamma_{0}\right) / s \Gamma_{0} \subseteq \Gamma_{0} / s \Gamma_{0}$, which is finite.

If we consider the smaller abelian subgroup $G_{0}=S^{-1} S$ of $G$ and let $\Gamma$ be the split extension of $N=S^{-1} \Gamma_{0}$ by $G_{0}$, the setting of Theorem 2.11 begins to appear, with $T=S^{-1}$ in this case.

\section{References}

[BC] J.B. Bost and A. Connes, Hecke Algebras, type III factors and phase transitions with spontaneous symmetry breaking in number theory, Selecta Math. (N.S.), 1(3) (1995), 411-457.

[B] B. Brenken, The local product structure of expansive automorphisms of solenoids and their associated $C^{*}$-algebras, Canad. J. Math., 48(4) (1996), 692-709.

[C] J. Cuntz, Simple $C^{*}$-algebras generated by isometries, Comm. Math. Phys., 57 (1977), 173-185.

[F] L. Fuchs, Infinite Abelian Groups, Vol. II, Academic Press, New York-London, 1973.

[LR] M. Laca and I. Raeburn, Semigroup crossed products and the Toeplitz algebras of nonabelian groups, J. Funct. Anal., 139(2) (1996), 415-440.

$[\mathrm{N}]$ A. Nica, $C^{*}$-algebras generated by isometries and Wiener-Hopf operators, J. Operator Theory, 27 (1992), 17-52.

[R] L. Rowen, Ring Theory, Vol. I, Academic Press, San Diego, 1988.

[ZS] O. Zairski and P. Samuel, Commutative Algebra, Vol. I, D. Van Nostrand Co., Toronto-New York-London, 1972.

Received August 5, 1996. The author acknowledges support, in connection with this research, from the Natural Sciences and Engineering Research Council of Canada and the Fields Institute for Research in the Mathematical Sciences.

UNiversity OF CALGARY

Calgary, AB T2N $1 \mathrm{~N} 4$

CANADA

E-mail address: bbrenken@ucalgary.ca 